University of South Carolina

Scholar Commons

Theses and Dissertations

$1-1-2013$

\title{
The Effects of A High Fat Diet On Mitochondrial Biogenesis and Inflammation In the Brain.
}

Lauren Suzanne Vervaecke

University of South Carolina

Follow this and additional works at: https://scholarcommons.sc.edu/etd

Part of the Exercise Science Commons

\section{Recommended Citation}

Vervaecke, L. S.(2013). The Effects of A High Fat Diet On Mitochondrial Biogenesis and Inflammation In the Brain.. (Master's thesis). Retrieved from https://scholarcommons.sc.edu/etd/1230

This Open Access Thesis is brought to you by Scholar Commons. It has been accepted for inclusion in Theses and Dissertations by an authorized administrator of Scholar Commons. For more information, please contact digres@mailbox.sc.edu. 
The Effects of a High Fat Diet on Mitochondrial Biogenesis and Inflammation in the Brain

By

Lauren S. Vervaecke

Bachelor of Science

St. Ambrose University, 2011

Submitted in Partial fulfillment of the Requirements

For the Degree of Master of Science in

Exercise Science

Arnold School of Public Health

University of South Carolina

2013

Accepted by:

J. Mark Davis, Major Professor

Angela Murphy, Reader

James Carson, Reader

Ray Thompson, Reader

Lacy Ford, Vice Provost and Dean of Graduate Studies 
CCopyright by Lauren S. Vervaecke, 2013

All Rights Reserved 


\section{DEDICATION}

I would like to dedicate my master's thesis to a few trusted individuals who have always been there for me in support of my education and life success. First, to my loving and supportive husband James; thank you for your kindness, understanding, and endless love through this challenging event. Your dedication to supporting my academic success has kept you by my side earning my masters degree right along with me. Together we make a great team and I could not have made it this far without you, congratulations on another hard earned degree.

Second, to my former advisor Dr. Laurent; your words have reached far beyond the classroom and have guided me along my academic journey. The challenging moments I have faced have been defeated by the lessons you have taught me and your inspiring motivation to go after what I want in life and to never give up.

And finally to Dr. Medema Johnson, your personal life experiences that you have shared with me have taught me so much about my own life and have positively affected the decisions I have made in my academic career. I cannot thank you enough for your constant encouragement and support.

Today I am on my way to a bright and successful future and it is all because of your love, support, and inspiring lessons of life. Thank you for everything! 


\begin{abstract}
INTRO: High fat diets (HFD) ingested over long periods of time have been shown to cause diet induced obesity leading to excessive adiposity and chronic low grade inflammation. Here we have shown how differing levels of saturated fats (SF), which are common storage fats, affect markers of inflammation and mitochondrial biogenesis. IL$1 \beta$ and TNF-alpha are pro-inflammatory cytokines that have been shown to increase with a HFD, while molecular signalers, PGC-1alpha, cyt c, and SIRT1, related to mitochondrial biogenesis have been shown to decrease. Despite the risks of consuming a diet high in SF's, consumers continue to buy energy dense foods that are well known to cause metabolic dysregulation. METHODS: Male C57BL/6 mice were randomized into groups (n=8/9) (AIN 76 A), (AIN 76 A mod), (6\% HFD), (12\% HFD), (24\% HFD). Mice were fed either HFD or LFD for 16 weeks. Body weights were measured weekly. At 20 weeks of age mice were sacrificed and brain parts (hypothalamus, hippocampus, and cerebellum) were isolated for mRNA analysis of two inflammatory cytokines (IL-1 $\beta$, TNF-alpha), and three molecular signalers (PGC-1alpha, SIRT1, and cyt c) using QTPCR. RESULTS: High fat feeding increased the accumulation of mass and obesogenic characteristics. Beginning at 12 weeks of age all HFD groups were different from control (AIN $76 \mathrm{~A})(\mathrm{P}<0.05)$. Beginning at 17 weeks of age the $12 \%$ HFD group was different from both the $6 \%$ and $24 \%$ HFD group. Gene expression of cyt $\mathrm{c}$ in the cerebellum increased in the $6 \%$ HFD relative to control $(\mathrm{P}<0.033)$ and gene expression of IL-1 $\beta$ in the hypothalamus increased in the $12 \% \mathrm{HFD}$
\end{abstract}


group relative to control ( $\mathrm{P}<0.031)$. CONLU: These findings suggest that diets high in saturated fat $(6 \%, 12 \%, 24 \%)$, but the same in total fat $(40 \%)$ leads to an increase in body weight and obesogenic characteristics, with the largest difference in the $12 \%$ HFD group. This was associated with increased IL- $1 \beta$ expression in the hypothalamus, while the $6 \%$ HFD was associated with an increase in cyt c expression suggesting changes in mitochondrial biogenesis in the cerebellum.

Obesity; Metabolism; Brain; Inflammation 


\section{TABLE OF CONTENTS}

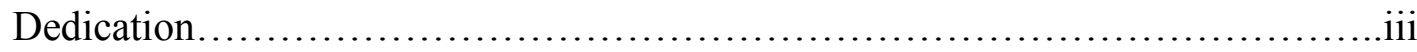

Abstract.....................................................................

List of Figures..........................................................

Chapter 1: The Effects of a High Fat Diet on Mitochondrial Biogenesis and Inflammation in the Brain

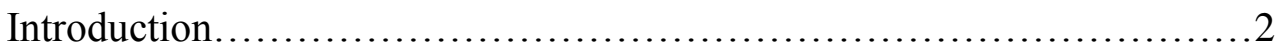

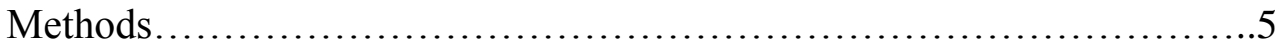

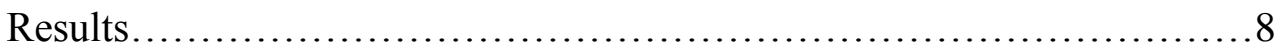

Discussion......................................................... 10

Chapter 2: Thesis Proposal

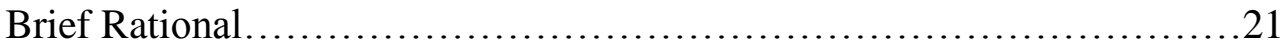

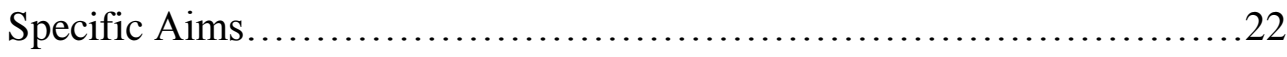

Proposed Model......................................................24

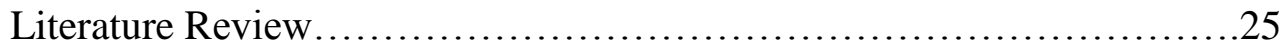

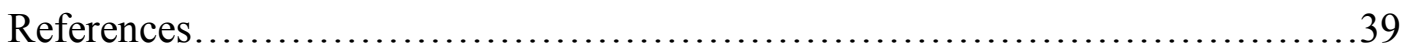




\section{LIST OF FIGURES}

Figure 1.1: Body weight.............................................. 15

Figure 1.2: PGC-1alpha gene expression................................... 16

Figure 1.3: SIRT1 gene expression.......................................17

Figure 1.4: Cytochrome C gene expression................................. 18

Figure 1.5: IL-1 beta gene expression.....................................19

Figure 1.6: TNF-alpha gene expression..................................20

Figure 2.1: Proposed Model.................................................24 
CHAPTER 1

THE EFFECTS OF A HIGH FAT DIET ON MITOCHONDRIAL BIOGENESIS AND INFLAMMATION IN THE BRAIN ${ }^{1}$

\footnotetext{
${ }^{1}$ L. Vervaecke, J.M. Davis, J. Carson, A. Murphy, and R. Thompson. To be submitted to Obesity.
} 


\section{INTRODUCTION}

Obesity has become a large scale epidemic spanning the globe affecting both adults and children causing severe health disparity and declines in quality of life. In the United States alone, more than one third of all Americans are overweight or obese and consume diets high in fat and excess calories (48). Many Americans suffer the ill-fated effects associated with obesity such as cardiovascular disease, atherosclerosis, hypertension, type 2 diabetes, fatty liver disease, and metabolic syndrome. The standard American diet consists of 52\% carbohydrate intake, $15 \%$ protein intake, and $33 \%$ fat intake, of which $12 \%$ consists of saturated fats (SF) $(23,26)$. High fat diets (HFD) ingested over long periods of time are well known to cause increased adiposity and chronic low grade inflammation $(37,53)$. SF's are well known storage fats that have been linked to diet induced obesity and excessive adiposity thereby up regulating proinflammatory pathways instigating insulin resistance and metabolic dysregulation (34). Despite the risks of consuming a diet high in SF's, consumers continue to buy these energy dense foods that are well known to cause metabolic dysregulation.

The majority of research that has assessed the effects of a HFD on mitochondrial biogenesis has been done in skeletal muscle and adipose tissue. We know that energy metabolism takes place in the mitochondria, the power house of the cell, and combines oxygen and desired fuel substrates, fats and carbohydrates, to be broken down into cellular energy in the form of adenosine triphosphate (ATP). These metabolic processes are regulated allosterically by adenosine monophosphate activated protein kinase

(AMPK). AMPK is a regulator of mitochondrial biogenesis and is the primary pathway regulating mitochondrial activity $(22,65)$. AMPK mediates actions of peroxisome proliferator activated receptor gamma co-activator alpha (PGC-1alpha) and silent 
information regulator one (SIRT1). SIRT1 acts by deacetylation of PGC-1alpha, a master regulator of mitochondrial biogenesis. SIRT1 has been shown to be a primary regulator of fatty acid oxidative metabolism and acts to protect against diet induced metabolic dysfunction (24).

Current research has demonstrated that chronic HFD's negatively affect mitochondrial capacity in skeletal muscle through dysfunctional enzymatic regulation and declines in the activity of molecular signalers $(1,58)$. HFD's have also been shown to induce oxidative stress and insulin insensitivity in peripheral tissues $(1,10,19,27,62$, 68). Chronic inflammation has been linked as a key component in diet induced obesity and has been shown to negate cellular interactions, insulin signaling, molecular signalers, and normal cellular functioning $(1,7,19)$. Two pro-inflammatory cytokines IL-1 $\beta$ and TNF-alpha are responsible for activating the $\mathrm{NF}_{\mathrm{k}} \mathrm{B}$ pathway inducing $\mathrm{M} 1$ macrophage infiltration (56). M1 macrophages activate pro-inflammatory cytokines and reactive oxygen species, which have been shown to cause mitochondrial collapse (25). A paucity of research has been done to elucidate the effects of HFD's on skeletal muscle; however, there is a gap in understanding if similar molecular dysregulation in peripheral tissues is observed within the brain and potentially the cause of mitchondrial dysregulation, oxidative stress, and therefore cognitive impairment and central fatigue.

The apogee of the central nervous system (CNS) is the brain, which is composed of highly complex centers that are innervated by neurons, which control a plethora of activities including movement, metabolism, memory, information processing, problem solving, mood and fatigue $(40,64)$. Dysfunctional neurogenic activity has been associated with fatigue, psychological impairments and cognitive disorders (28, 43, 64, 
70). Alzheimer's disease and age related dementia are two disorders that have been correlated with diet induced obesity and have been the main focus of HFD studies in the brain $(43,70)$. Diets high in SF's and cholesterol have demonstrated declines in the integrity of the blood brain barrier and severe gliosis throughout the hippocampus (21). Over nutrition has also been linked to increased inflammation in hypothalamic neurons (66). Recently it has been suggested that nutrient regulation by SIRT1 with a HFD was reduced in the hippocampus adversely affecting memory function $(28,72)$. Mitochondrial swelling has also been observed with an acute HFD and is believed to cause mitchondrial dysregulation; however, molecular signalers and specific mitochondrial markers were not analyzed (54).

Skeletal muscle has been copiously researched regarding changes in mitochondrial capacity and inflammation with a HFD, but minute research is available observing if similar changes take place within the brain. Mitochondrial signalers have been shown to be important mediators in skeletal muscle metabolism, exercise performance, delayed onset of fatigue, and endurance capacity, but are peripheral and central activity similarly affected with a HFD? The purpose of this 16 week study was to determine the effect of diets high in saturated fat $(6 \%, 12 \%, 24 \%)$, but the same in total fat $(40 \%)$, on mitochondrial biogenesis and inflammation in the brain. Previous research in our lab has shown that a HFD of $12 \% \mathrm{SF}$, the most similar to the standard American diet, showed the greatest amount of adiposity, inflammation, and metabolic dysregulation in peripheral tissues (17). We hypothesize that a HFD consisting of $12 \%$ SF will reduce mitochondrial biogenesis and increase inflammation in the brain. 


\section{METHODS}

\section{Animals}

Male C57BL/6 mice were bred and cared for in the animal facility at the University of South Carolina. They were housed, four-five per cage, maintained on a 12:12-h light-dark cycle in a low stress environment $\left(22^{\circ} \mathrm{C}, 50 \%\right.$ humidity, low noise $)$ and given food and water ad libitum. The Institutional Animal Care and Usage Committee of the University of South Carolina approved all experiments.

\section{Diets}

Experiment 1: At four weeks of age, mice were randomly assigned to 1 of 5 treatment diets ( $\mathrm{n}=8-9 /$ group): two control diets (CDs) (AIN-76A-1.6\%-SF) (AIN-76AMod-1.7\%-SF) and three HFDs (6\%-SF, 12\%-SF, and 24\%-SF) (BioServ, Frenchtown, NJ).The percentage of calories provided by each of the three macronutrients, the ratio of polyunsaturated: monounsaturated fatty acids (PUFA:MUFA), and the ratio of omega6:omega-3 fatty acids were identical for the HFDs and were designed to be similar to the standard American diet $(26,61)$. The second CD (AIN-76A-Mod) was used in order to match the PUFA: MUFA and omega-6: omega-3 ratios of the HFDs.

Experiment 2: At four weeks of age mice were randomly assigned to 1 of 2 treatment diets (n=5-9/group): HFD (12\%) or CD (AIN-76A) (BioServ, Frenchtown, NJ). The percentage of calories provided by each of the three macronutrients, the ratio of PUFA: MUFA and the ratio of omega-6: omega-3 fatty acids were designed to be similar to the standard American diet $(26,61)$. 


\section{Body weights, food intake, and body composition}

Body weight (BW) and food intake were monitored weekly (experiment 1 and 2).

Body composition was assessed every four weeks (weeks 4, 8, 12, 16, and 20)

(experiment 1 only). For this procedure, mice were placed under brief anesthesia

(isoflurane) and were assessed for lean mass (LM), fat mass (FM), and body fat

percentage (BF \%) via dual-energy x-ray absorptiometry (DEXA) (Lunar PIXImus,

Madison, WI).

\section{Tissue collection}

At 20 weeks of age, mice were sacrificed for tissue collection. The brain was dissected out into 7 sections: brain stem, cerebellum, cortex, frontal lobe, hippocampus, midbrain, and the hypothalamus. All tissues extracted were immediately snap-frozen in liquid nitrogen and stored at $-80^{\circ} \mathrm{C}$. Blood was collected from the inferior vena cava, and centrifuged at 4,000 rpm for $10 \mathrm{~min}$ at $4{ }^{\circ} \mathrm{C}$. Plasma was aliquoted and stored at $-80^{\circ} \mathrm{C}$.

\section{mRNA expression}

Brain tissue $(n=8 / 9)$ were homogenized under liquid nitrogen with a Polytron (PT3100; Kinematica, Lucerne, Switzerland), and the quanidine thiocyanate method with TRIzol reagent (Life Technologies, GIBCOBRL, Carlsbad, CA) was used to extract total RNA. The extracted RNA (2.5ul of sample) was dissolved in diethylpyrocarbonate treated water and quantified spectrophotometrically at 260nm wavelength. The 260:280 ratio was used to determine the quality of RNA; RNA with a ratio $>1.6$ was included in the analysis. RNA was reverse transcribed into cDNA in a 50ul reaction volume containing 19.25ul RNA (1.5ug) in RNase-free water, 5ul 10X RT buffer, $11 \mathrm{ul} 25 \mathrm{mM}$ $\mathrm{MgCl}_{2}, 10 \mathrm{ul}$ deoxy-NTP mixture, $2.5 \mathrm{ul}$ random hexamers, 1ul RNase inhibitor, and 
1.25ul multiscribe reverse transcriptase (50U/ul). Reverse transcription was completed at $25^{\circ} \mathrm{C}$ for $10 \mathrm{~min}, 37^{\circ} \mathrm{C}$ for $120 \mathrm{~min}$, and $85^{\circ} \mathrm{C}$ for $5 \mathrm{~min}$, followed by a quick chilling on ice. Samples were stored at $-20^{\circ} \mathrm{C}$ until subsequent amplification.

\section{Quantification of real time PCR}

Real time PCR analysis was carried out per manufacturer's instructions (Applied Biosystems, Foster City, CA) using TaqMan Gene Expression Assays. Amplification of DNA as performed in 12.5ul TaqMan Universal PCR Master Mix (AmpliTaq Gold DNA Polymerase, Passive Reference 1. Buffer, dNTPs; AmpEase UNG), 1ul cDNA, 9ul RNase-free water, 1.25ul 18S primer (VIC), and 1.25ul primer (FAM) (for endogenous references and target gene) in a final volume of 25ul/well. A MicoAmp 96 well reaction plate was loaded with samples and run using an ABI Sequence Detection System. Following $2 \mathrm{~min}$ at $50^{\circ} \mathrm{C}$ and $10 \mathrm{~min}$ at $95^{\circ} \mathrm{C}$, plates were coamplifed by 40 repeated cycles, with one cycle consisting of a 15 -s denaturing step at $95^{\circ} \mathrm{C}$ and a $1 \mathrm{~min}$ annealing/extending step at $60^{\circ} \mathrm{C}$. The data were analyzed by ABI software using the cycle threshold $\left(\mathrm{C}_{\mathrm{T}}\right)$, or the value calculated and based on the time at which the reported fluorescent emission increases beyond a threshold level citing the cycle number at which the cDNA amplification was first detected.

\section{Quantification of mRNA expression}

PGC-1alpha, SIRT1, cyt c, IL-1 $\beta$, TNF-alpha expression were calculated using a single calibrator sample to compare every known samples gene expression against. Breifly, $\Delta \mathrm{C}_{\mathrm{T}}\left[\mathrm{C}_{\mathrm{T}}(\mathrm{FAM})-\mathrm{C}_{\mathrm{T}}(\mathrm{VIC})\right]$ was calculated for each sample and the average $\Delta \mathrm{C}_{\mathrm{T}}$ of the control mice was then used as the calibrator sample. Initial exclusion criteria consisted of FAM $C_{T} \geq 40$ and VIC $C_{T} \leq 23$ and results showed all samples $C_{T}$ values 
were $\mathrm{VIC} \mathrm{C}_{\mathrm{T}} \leq 15$, and $\mathrm{FAM} \mathrm{C}_{\mathrm{T}} \geq 33$. This analysis and quantification method has been shown to yield similar results as the standard curve method.

\section{Statistical analysis}

Data regarding mitochondrial markers and inflammatory markers were analyzed using a one-way analysis of variance (ANOVA) to observe changes between all groups. Student Newman Keuls post hoc test was used to make comparisons where appropriate. All data were analyzed using commercial software (SigmaStat, SPSS, Chicago, IL). Statistical significance was set with an alpha value of $\mathrm{P}<0.05$. Data are presented as mean $( \pm$ SEM).

\section{RESULTS}

\section{Diets}

Mice were housed 4-5/ cage and caloric intake was assessed by the total amount of food eaten divided by the number of mice per cage. Food and water intake was measured weekly. In general, all HFD groups consumed similar food and water intake throughout the 16 weeks of feeding relative to control.

\section{Body weights}

Body weights were measured weekly to determine changes in total body weight in regards to the differing ratios of saturated fat in the diet [Figure 1.1]. Body weight measurements taken show that HFD increases the accumulation of mass and obesogenic characteristics. Beginning at 12 weeks of age all HFD groups were different from control (AIN $76 \mathrm{~A})(\mathrm{P}<0.05)$. Beginning at 13 weeks of age all HFD groups were different from control (AIN 76 A mod) $(\mathrm{P}<0.05)$. By weeks 17-20 the 12\% HFD group was significantly greater in weight from both 6\% HFD and 24\% HFD $(\mathrm{P}<0.05)$. 


\section{Gene expression for mitochondrial biogenesis}

Gene expression of SIRT1, PGC-1alpha, and cyt c were examined in the hypothalamus, hippocampus, and cerebellum portions of the brain. PGC-1alpha expression showed no differences between groups in all three portions of the brain [Figure 1.2]. SIRT1 expression was measured in the hippocampus and cerebellum, but not in the hypothalamus due to limited reagents. Despite changes observed in the literature with HFD, no significant differences were found between groups [Figure1.3]. When examining cyt c, we found an increase in gene expression within the 6\% HFD relative to control (AIN $76 \mathrm{~A})(1.64 \pm 0.28)$ vs. $(1.00 \pm 0.07)(\mathrm{P}<0.033)$, but no differences were seen in cyt c gene expression in the 12\% and 24\% HFD relative to control [Figure 1.4]. We measured two markers of mitochondrial biogenesis including SIRT1 and PGC1alpha, which are molecular signalers involved in stimulating mitochondrial biogenesis and also cyt c, which is an enzyme in the electron transport chain associated with mitochondrial adaptation. Overall it was shown that the metabolic signalers associated with mitochondrial biogenesis were not significantly altered by 16 weeks of high fat feeding, however, unexpectedly, cyt c was increased with high fat feeding only in the cerebellum.

\section{Gene expression for inflammation}

Gene expression of IL-1 $\beta$ and TNF-alpha were examined in the hypothalamus, hippocampus, and cerebellum regions of the brain. TNF-alpha expression showed no significant group differences in any of the three brain regions [Figure1.6]. However, IL$1 \beta$ a mediator of TNF-alpha through IL-1 receptor signaling, which activates the $\mathrm{NF}_{\mathrm{k}} \mathrm{B}$ 
pathway, was found to be significantly higher in the 12\% HFD group compared to control (AIN 76 A) $(2.108 \pm 0.330)$ vs. $(1.00 \pm 0.06)(\mathrm{P}<0.031)$ [Figure 1.5].

\section{DISCUSSION}

It has been well established that diet induced obesity by HFD is commonly associated with increased risk of cardiovascular disease, diabetes, and many other pathologies due to the accumulation of adipose tissue leading to chronic inflammation, thereby causing metabolic dysregulation. Skeletal muscle has been copiously researched to elucidate the extent of oxidative stress, fatigue, inflammation, and metabolic dysregulation, however, the brain is still poorly understood in regards to these same developments. The purpose of this 16 week study was to determine the effect of diets high in $\mathrm{SF}(6 \%, 12 \%, 24 \%)$, but the same in total fat $(40 \%)$, on mitochondrial biogenesis and inflammation in the brain. In general all HFD groups increased in size and body mass; however, the 12\% HFD group showed an increase in mass independent of the $6 \%$ and 24\% HFD group [Figure 1.1]. Previous research in our lab has shown that a diet of $12 \% \mathrm{SF}$, the most similar to the standard American diet, observed the greatest amount of adiposity, inflammation, and metabolic dysregulation in peripheral tissues (17). Despite similar characteristics in function between central and peripheral tissues, the brain shows disparate outcomes in inflammation and mitochondrial capacity with a HFD. Our primary finding was that the $12 \%$ HFD causes an increase in IL-1 $\beta$ expression in the hypothalamus; a primary regulator of metabolism.

The first aim of our study was to elucidate how differing ratios of SF $(6 \%, 12 \%$, 24\%) affect mitochondrial biogenesis in the brain. To do this we examined changes in expression of two molecular signalers, SIRT1 and PGC-1alpha, and cyt c, an important 
enzyme in the electron transport chain. Several studies have worked to examine the effects of a diet high in fat on mitochondrial capacity in the brain; however, no studies have been completed to determine, more specifically, how diets high in SF affect similar variables. Brain HFD research has been primarily focused on Alzheimer's disease and age related dementia with the aim to determine how chronic ingestion of a HFD affects memory consolidation and information processing. Recently it was shown that 23 weeks of high fat feeding with $60 \% \mathrm{kcal}$ coming from fat causes a decline in SIRT1 gene expression in the hippocampus suggesting a decrease in mitochondrial biogenesis and a subsequent decline in hippocampus-dependent memory (28). In discordance, our results, after 16 weeks of high fat feeding with $40 \% \mathrm{kcal}$ coming from fat and differing ratios of SF $(6 \%, 12 \%, 24 \%)$, show no change in SIRT1 across all groups [Figure 1.3]. Based on previous research in our lab, we expected to see a decline in SIRT1 gene expression within the $12 \% \mathrm{HFD}$, as we have shown it be the most metabolically dysregulating in peripheral tissues (17). However, it is difficult to compare current literature citing a change in SIRT1 gene expression due to differences in study duration; 16 versus 23 weeks, and diet composition; $40 \%$ versus $60 \% \mathrm{kcal}(28)$.

We also assessed central mitochondrial biogenesis by examining changes in the expression of PGC-1alpha. Our results show no change in PGC-1alpha with a 16 week HFD [Figure 1.2]. Extensive literature is available regarding changes in PGC-1alpha in skeletal muscle with a HFD; however, there are few studies that have looked similar changes in the brain. Richardson et al. 2005 shows a decline in PGC-1alpha expression in muscle tissue post 2 day lipid infusion (20\% triglyceride emulsion, $30 \% \mathrm{kcal}$ ) in human subjects (58). Although an 8 week intervention in mice suggests that a high fat feeding 
with $47 \% \mathrm{kcal}$ coming from fat, does not impede mitochondrial biogenesis, but in fact enhances it with an increase in PGC-1alpha expression (29). In accordance with our findings in the brain, Draznin et al. 2012 found no change in PGC-1alpha or other mediators of mitochondrial biogenesis in skeletal muscle with a HFD (50\% kcal) (16), suggesting that duration and diet composition are major limitations to observing mitochondrial changes with the induction of a HFD.

Cyt $\mathrm{c}$ was the final marker of examination to determine changes in mitochondrial biogenesis. Cyt $\mathrm{c}$ is a component of the electron transport chain that is commonly used to observe mitochondrial adaptations (39). Our results show an increase in cyt c expression within the cerebellum of the $6 \%$ HFD group compared to control [Figure 1.4], suggesting that mitochondrial biogenesis was increased with a HFD. However, we believe that this may be an extraneous effect of medium versus long chain fatty acid breakdown, due to the fact that no changes were observed in cyt $\mathrm{c}$ in the higher saturated fat groups; the $12 \%$ HFD and the 24\% HFD. Medium chain fatty acids are quickly transported and oxidized within the body compared to long chain fatty acids, which are less efficiently oxidized and more likely to be stored as adipose tissue (46).

There is a known link between diet induced obesity and systemic inflammation and it has been suggested that central inflammation may be related to the development of Alzheimer's disease and age related dementia by neuronal dysfunction. The final aim of our study was to examine how diets high in SF $(6 \%, 12 \%, 24 \%)$ affect inflammation in various regions of the brain. To our knowledge only one published study has reported changes in the brain due to a diet high in SF. Findings describe alterations in the integrity of the blood brain barrier of the hippocampus with increased microgliosis in HFD fed 
mice (45\% kcal) (21). In addition Thaler et al. 2012 has shown that diet induced obesity $(60 \% \mathrm{kcal})$ causes inflammation in the brain with only 4 days of high fat feeding, where as it takes weeks to observe inflammation in muscle $(66,67)$. Our results show an increase in IL-1 $\beta$ in the hypothalamus in the $12 \%$ HFD group after 16 weeks of high fat feeding, however, no changes were seen in the hippocampus or the cerebellum [Figure1.5].

IL- $1 \beta$ mediates the up regulation of many other inflammatory cytokines like TNFalpha through IL-1 receptor signaling subsequently activating the $\mathrm{NF}_{\mathrm{k}} \mathrm{B}$ pathway (45). $\mathrm{NF}_{\mathrm{k}} \mathrm{B}$ works to increase systemic lipolysis and glycogenolysis thereby increasing free fatty acids and glucose in the blood that chronically results in insulin resistance. Despite changes in IL-1 $\beta$ expression in the hypothalamus, we did not observe changes in TNFalpha expression across all groups in any of the examined brain parts. With regards to the development of severe pathology related to diet induced obesity, such as metabolic syndrome, the $\mathrm{NF}_{\mathrm{k}} \mathrm{B}$ pathway is activated by low grade inflammation and chronically orchestrates M1 machrophage infiltration that increases pro-inflammatory cytokines such as TNF-alpha and IL-1 $\beta$, as well as reactive oxygen species (56). This positive feedback loop of inflammation has been shown to cause mitochondrial damage and collapse as well as cellular apoptotic responses (25). The severity of the injury will dictate the time frame in which inflammatory cytokines are up regulated, some injuries necessitate more time than others.

In conclusion, this is the first study to examine the effects of differing levels of SF's on mitochondrial biogenesis and inflammation in the brain. We can conclude that diets high in SF $(6 \%, 12 \%, 24 \%)$, but the same in total fat $(40 \%)$ lead to an increase in 
body weight and obesogenic characteristics, with the largest difference in the 12\% HFD group, which was associated with increased hypothalamic IL-1 $\beta$ expression, a marker of inflammation. The 6\% HFD group was associated with changes in mitochondrial biogenesis in the cerebellum. It should be taken into consideration that in this study and others, these responses are highly dependent upon the amount of SF in the diet and total calories from fat. Limitations in this study include duration of the HFD and diet composition. This study has provided a basic understanding of how diets high in SF affect mitochondrial biogenesis and inflammation in the brain, future research is warranted to determine specific timelines in which mitochondrial biogenesis is affected along with changes in the normal inflammatory response. 


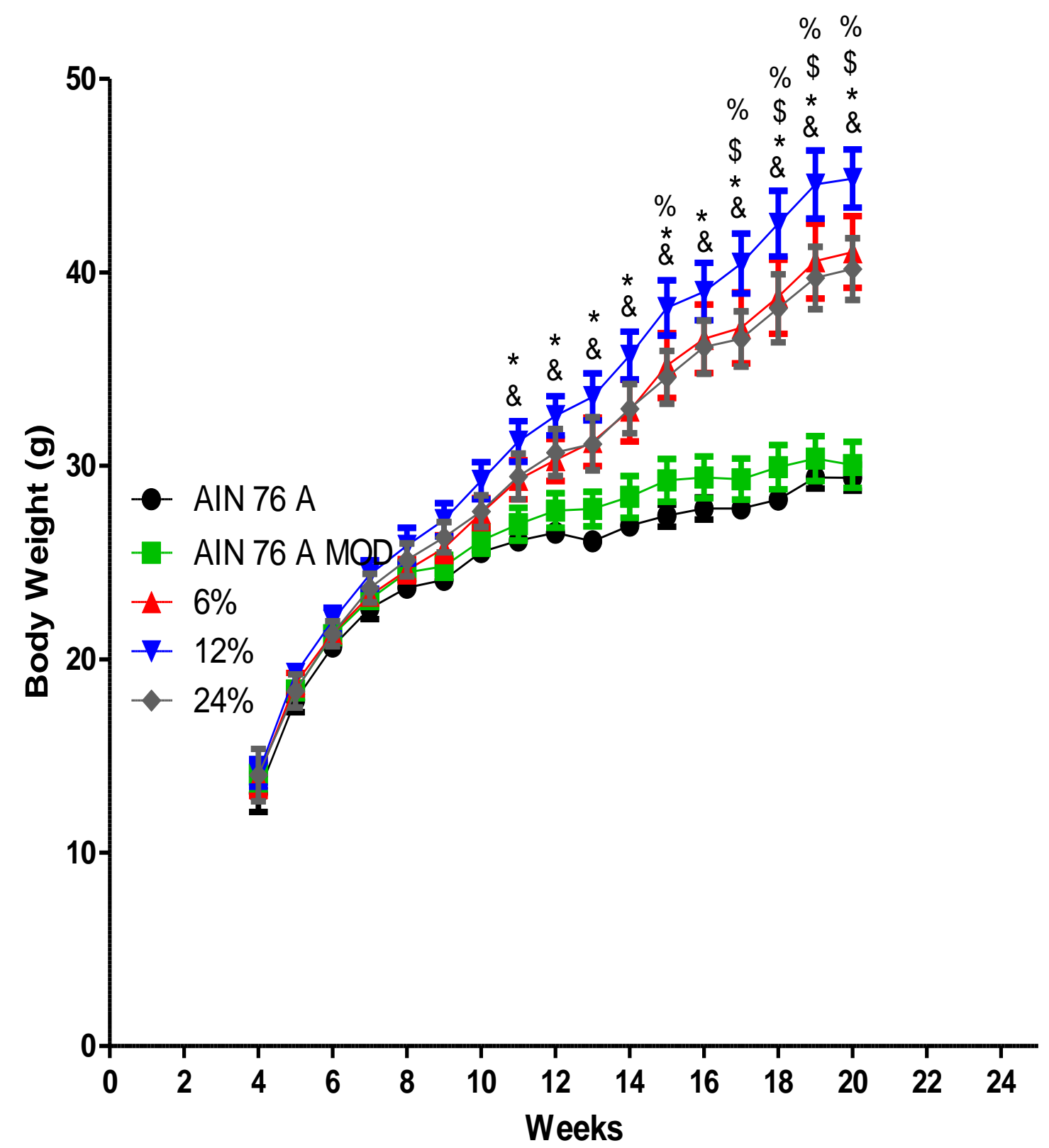

Figure 1.1: The effect of diets high in saturated fat on body weight. Body weights were measured weekly and are reported from 4 weeks of age to 20 weeks of age. AIN 76 A (n=8), AIN 76 A mod (n=9), 6\% HFD (n=9), 12\% HFD (n=9), and 24\% HFD (n=8). \&Significantly different from AIN 76 A mod, P <0.05 (weeks 11-12, 12\% HFD only) (weeks 13-20, 24\%, 12\%, 6\% HFD). *Significantly different from AIN 76 A, P<0.05 (week 11, 12\% HFD only) (weeks 12-20, 24\%, 12\%, 6\% HFD). \% Significantly different from $6 \%$ HFD, $\mathrm{P}<0.05$ (weeks 15, 17-20, 12\% HFD only). \$Significantly different from $24 \%$ HFD, $\mathrm{P}<0.05$ (weeks 17-20, 12\% HFD only). 

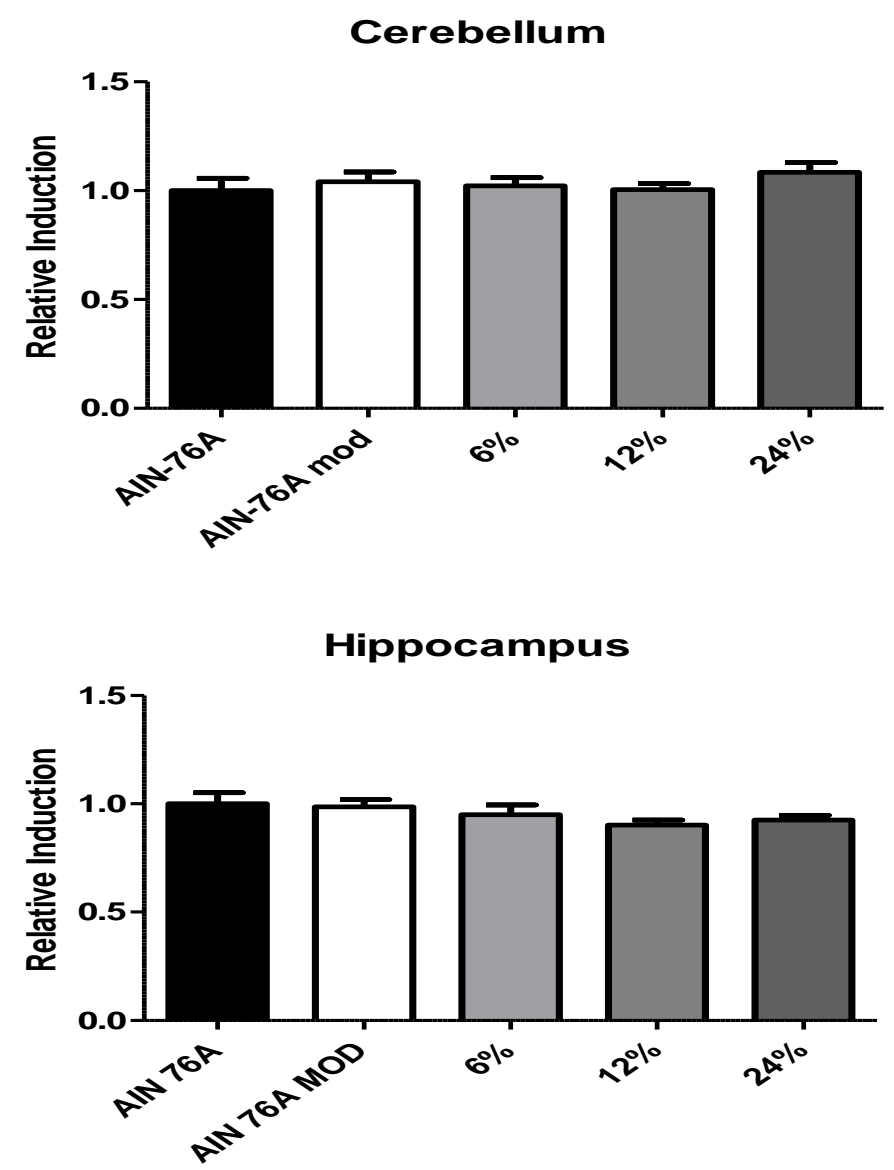

Hypothalamus

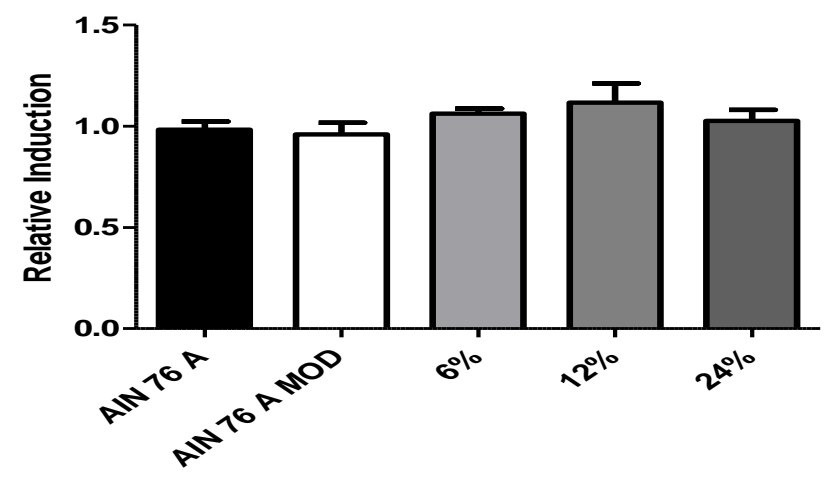

Figure 1.2: The effect of saturated fat on PGC-1alpha gene expression in HFD fed mice. Gene expression (PGC-1alpha) for all groups is reported from the time of sacrifice, 20 weeks of age. AIN 76 A (Con $n=6)$, AIN 76 A $\bmod (\operatorname{Con} n=6), 6 \%$ HFD $(6 \% n=6)$, $12 \%$ HFD ( $12 \% \mathrm{n}=6), 24 \%$ HFD ( $24 \% \mathrm{n}=6)$. No significant differences were found. Values are reported as mean \pm SE. 


\section{Cerebellum}

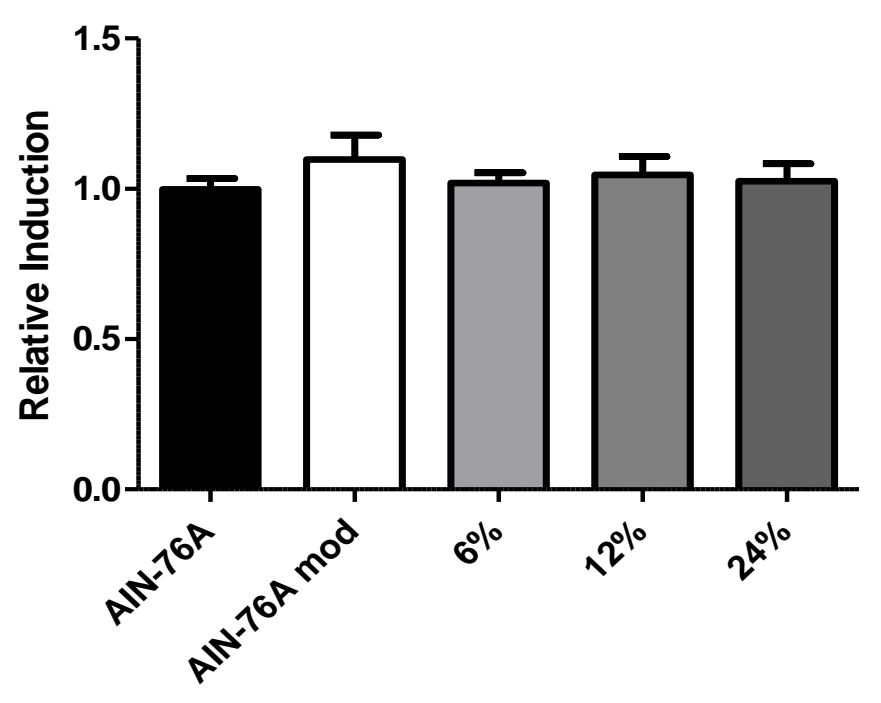

Hippocampus

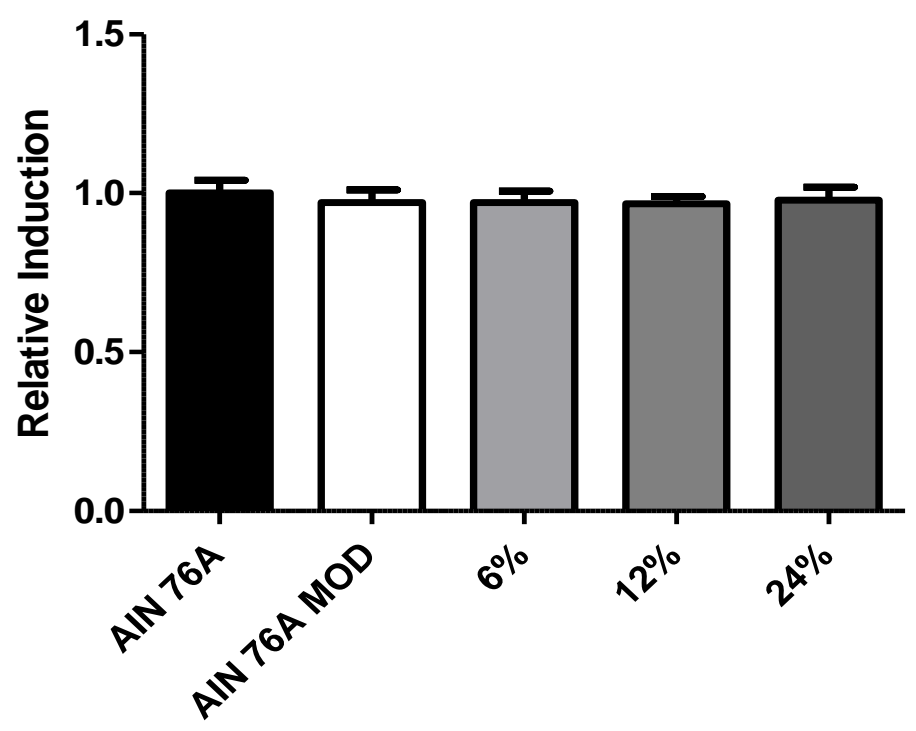

Figure 1.3: The effect of saturated fat on SIRT1 gene expression in HFD fed mice. Gene expression (SIRT1) for all groups is reported from the time of sacrifice, 20 weeks of age. AIN 76 A (Con $n=6)$, AIN 76 A mod (Con $n=6), 6 \%$ HFD (6\% $n=6), 12 \%$ HFD $(12 \% \mathrm{n}=6), 24 \%$ HFD $(24 \% \mathrm{n}=6)$. No significant differences were found. Values are reported as mean $\pm \mathrm{SE}$. 

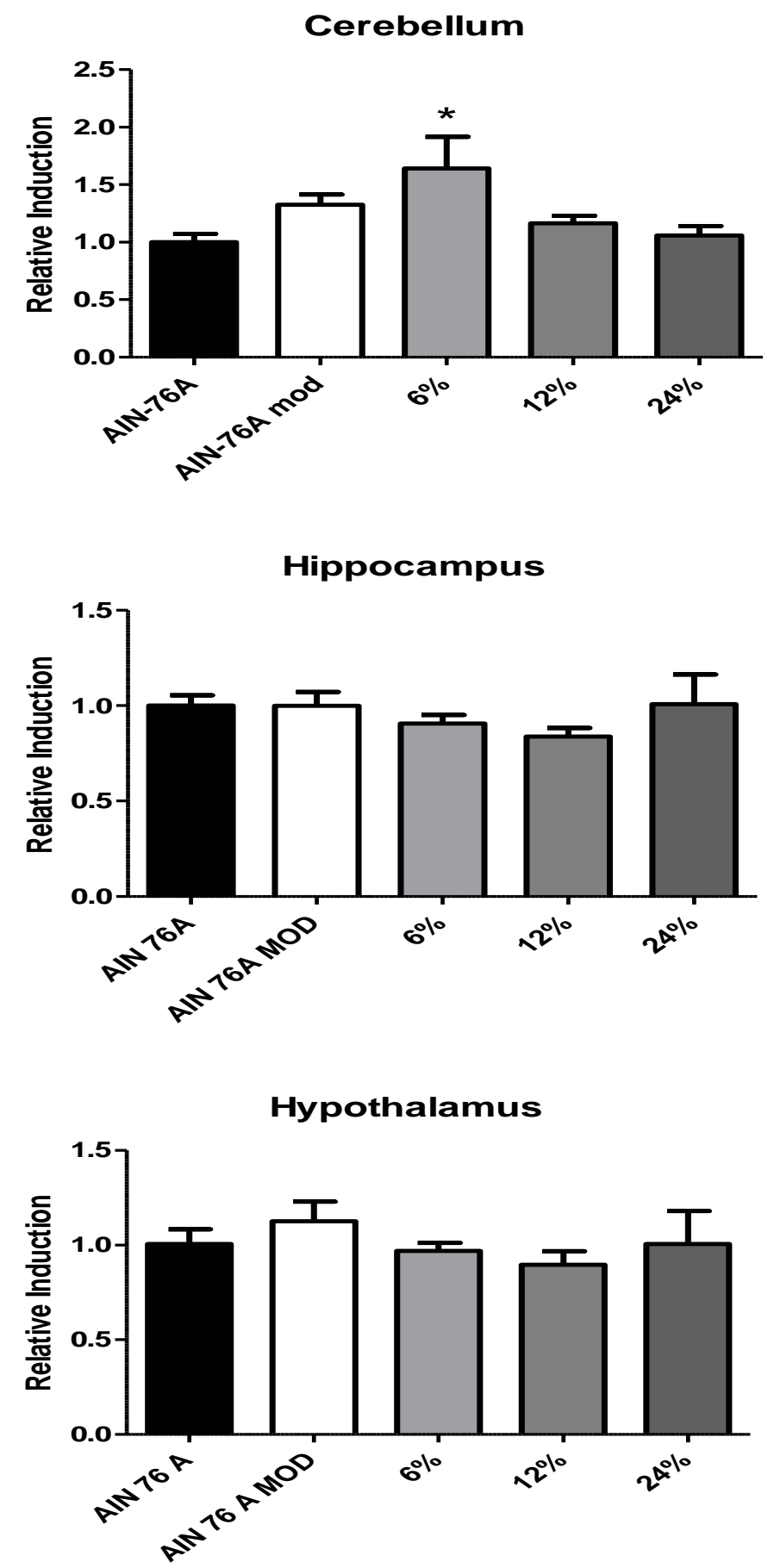

Figure 1.4: The effect of saturated fat on CYT C gene expression in HFD fed mice. Gene expression (cyt c) for all groups is reported from the time of sacrifice, 20 weeks of age. AIN 76 A (Con $n=6)$, AIN 76 A mod (Con $n=6), 6 \%$ HFD (6\% n=6), 12\% HFD $(12 \% \mathrm{n}=6), 24 \%$ HFD $(24 \% \mathrm{n}=6)$. * Significantly different from the control diet (AIN 76 A), $\mathrm{P}<0.05$. Values are reported as mean $\pm \mathrm{SE}$. 

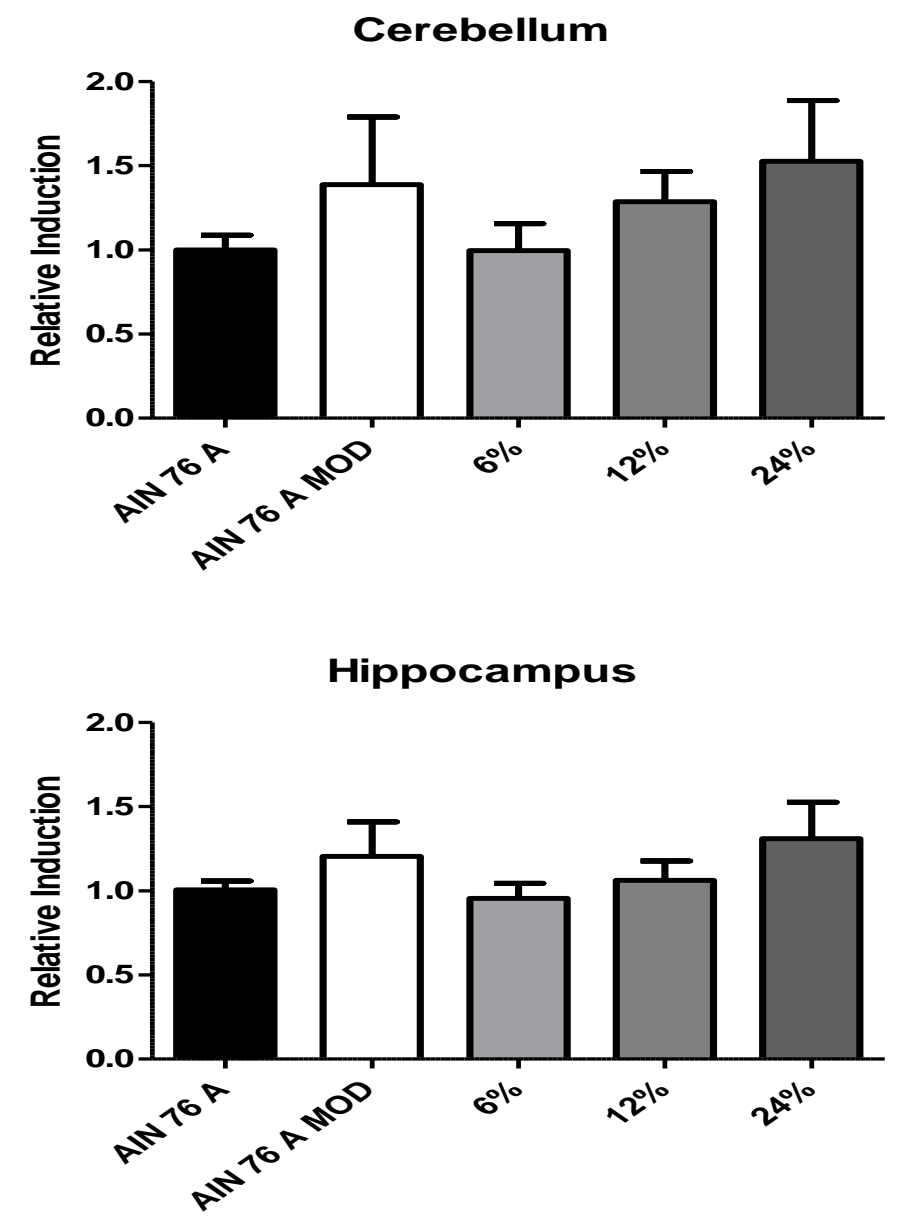

Hypothalamus

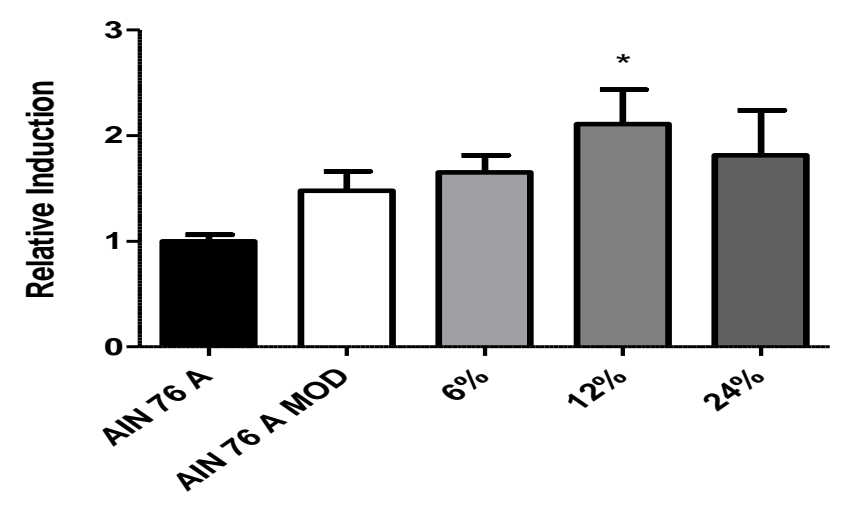

Figure 1.5: The effect of saturated fat on IL-1beta gene expression in HFD fed mice. Gene expression (IL-1beta) for all groups is reported from the time of sacrifice, 20 weeks of age. AIN 76 A (Con n=6), AIN 76 A mod (Con $n=6), 6 \%$ HFD (6\% n=6), 12\% HFD $(12 \% \mathrm{n}=6), 24 \%$ HFD $(24 \% \mathrm{n}=6)$. *Significantly different from control diet (AIN $76 \mathrm{~A})$, $\mathrm{P}<0.05$. Values are reported as mean $\pm \mathrm{SE}$. 

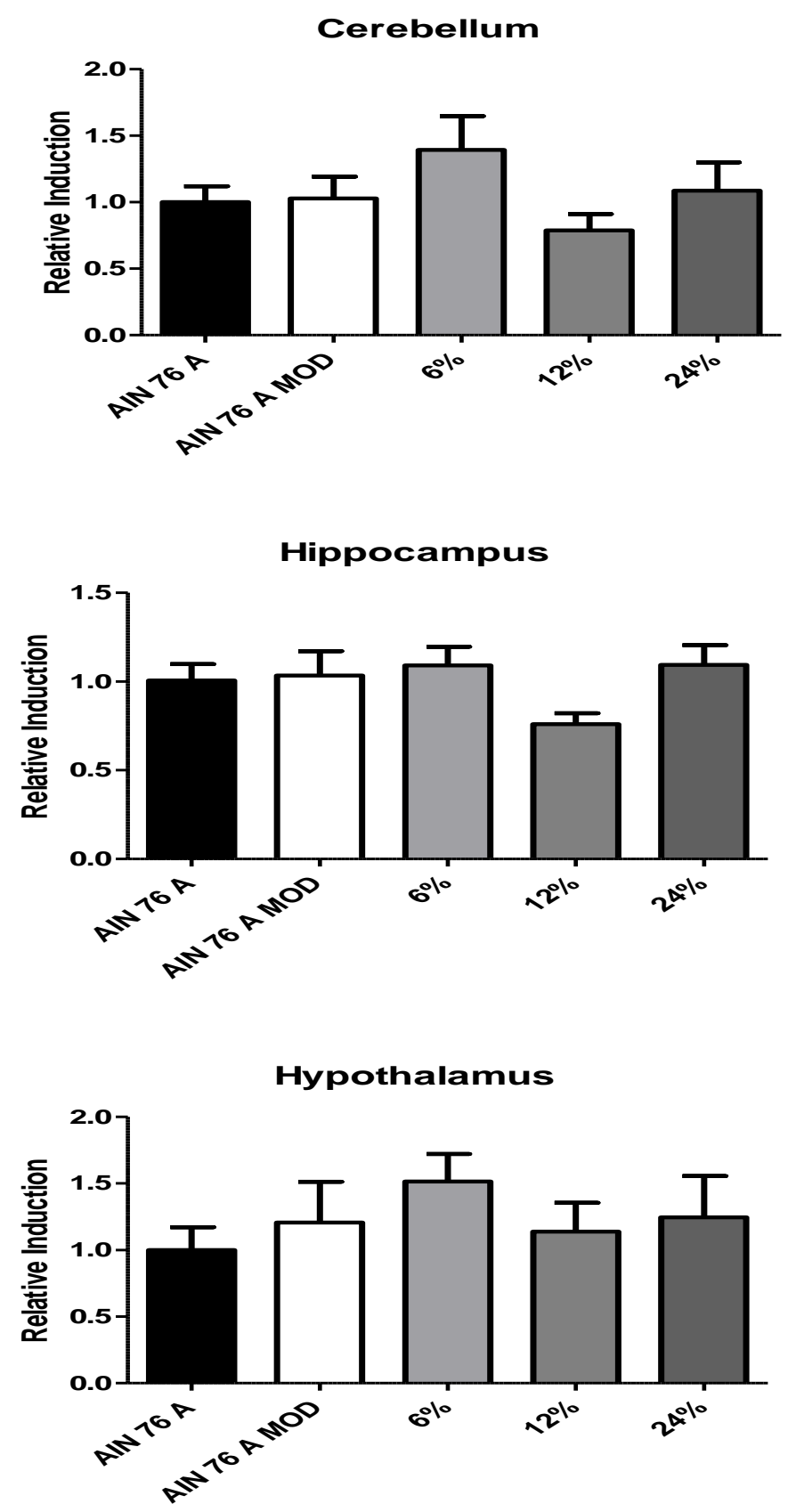

Figure 1.6: The effect of saturated fat on TNF-alpha gene expression in HFD fed mice. Gene expression (TNF-alpha) for all groups is reported from the time of sacrifice, 20 weeks of age. AIN 76 A (Con n=6), AIN 76 A mod (Con $n=6), 6 \%$ HFD (6\% n=6), $12 \%$ HFD $(12 \% \mathrm{n}=6), 24 \%$ HFD ( $24 \% \mathrm{n}=6)$. No significant differences were found. Values are reported as mean $\pm \mathrm{SE}$. 


\section{CHAPTER 2}

\section{THESIS PROPOSAL}

\section{BRIEF RATIONAL}

More than one third of all Americans are overweight or obese and consume diets high in saturated fat, many of which suffer the ill-fated effects associated with obesity such as hypertension, type 2 diabetes, and metabolic dysfunction. Energy metabolism in the form of cellular respiration takes place in the mitochondria, the power house of the

cell, and combines oxygen and fuel sources such as fats, carbohydrates, and proteins to be catabolized into cellular energy in the form of adenosine triphosphate (ATP). All metabolic functions require the use of ATP to sustain activity and to carry out daily processes. Muscle being a highly plastic organ can store fats and carbohydrates as intramuscular triglycerides (IMTG) and glycogen. The standard American diet consists of $52 \%$ carbohydrate intake, $15 \%$ protein intake, and $33 \%$ fat intake of which $12 \%$ consists of saturated fat. Sparks et al. 2006 has elucidated that diets high in saturated fat and overall total fat have negative effects on energy metabolism in skeletal muscle mitochondria related to dysfunctional enzymatic regulation and mitochondrial biogenesis, as well as gene expression controlling glucose metabolism and storage. Chronic inflammation related to obesity has also been shown to negate cellular interactions, insulin signaling, enzymatic regulators, and normal cellular functioning. However, minute research has been completed observing if similar changes take place within the brain. 
The apogee of the central nervous system (CNS) is the brain, which is composed of highly complex centers that control processes like movement, sight, hearing, emotions, and metabolism. The hypothalamus, hippocampus, motor cortex, cerebellum, and brain stem are the primary controllers of these bodily functions. Glucose being the primary fuel source within the CNS is responsible for neurogenic activity. Astrocytes are glial cells that are positioned between blood vessels and neurons that control energy metabolism in the brain by feeding glucose to neurons. Neurons within the CNS control a plethora of activities including attention, memory, information processing, problem solving, impaired mood, and fatigue. Dysfunctional neurogenic activity has been associated with fatigue, psychological impairments, and cognitive disorders related to malfunctions within the astrocytes in the hypothalamus and the hippocampus, but what is causing this.

To date skeletal muscle has been highly researched regarding changes in mitochondrial activity and glycogen storage with the manipulation of a high fat diet, but minute research has been completed observing if similar changes take place within the brain. Mitochondria and glycogen, as well as their subsequent regulators, have been shown to be important mediators in skeletal muscle regarding energy metabolism and endurance capacity, but are peripheral and central activity similarly affected with a high fat diet?

\section{PURPOSE AND SPECIFIC AIMS}

The purpose of this 16 week study was to determine the effect of diets high in saturated fat $(6 \%, 12 \%, 24 \%)$, but the same in total fat (40\%), on mitochondrial biogenesis and inflammation in the brain. Previous research in our lab has shown that a diet of $12 \%$ saturated fat, the most similar to the standard American diet, observed the 
greatest amount of adiposity, inflammation and metabolic dysregulation in peripheral tissues.

Aim1: To determine the effects of a high fat diet $(6 \%-\mathrm{SF}, 12 \%-\mathrm{SF}, 24 \%-\mathrm{SF})$ and (40\%) total fat on mitochondrial biogenesis in the brain.

We hypothesize that a high fat diet of 12\%-SF will reduce mitochondrial biogenesis in the brain. We will use RNA isolation and real time PCR to measure gene expression of PGC-1alpha, SIRT1, and cytochrome c in the hippocampus, hypothalamus, and cerebellum.

Aim 2: To determine the effects of a high fat diet (12\%-SF) and (40\%) total fat on inflammation in the brain.

We hypothesize that a high fat diet of 12\%-SF will increase inflammation in the brain. We will use RNA isolation and real time PCR to measure gene expression of IL-1B and TNF-alpha in the hippocampus, hypothalamus, and cerebellum. 


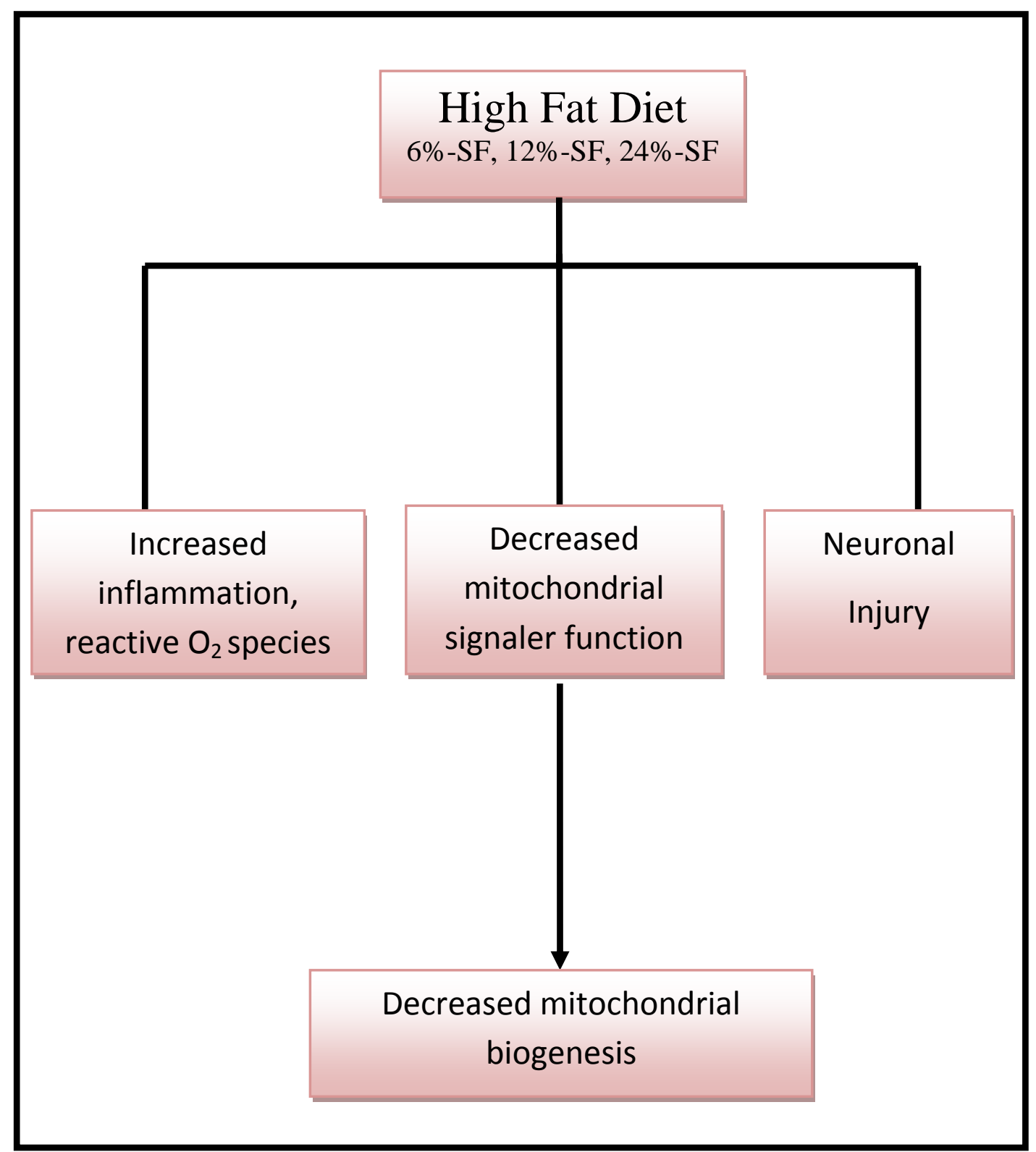

Figure 2.1: This study is aimed to uncover the proposed model of a diet high in saturated fat $(6 \%, 12 \%, 24 \%)$, but the same in total fat $(40 \%)$ on the effects of mitochondrial biogenesis and inflammation in the brain. Based on previous research it is theorized that the $12 \%$ saturated fat diet, the diet most similar to the standard American diet, will observe the most mitochondrial dysfunction related to changes mitochondrial signaler regulation in combination with increased inflammation and neuronal injury therefore potentiating CNS impairment. 


\section{LITERATURE REVIEW}

\section{Energy Metabolism}

Metabolism is the summation of all cellular work occurring at random within the body, but more specifically, energy metabolism is a cyclic response of turnover and synthesis to sustain usable energy (40). Cellular energy can be derived in two ways: through aerobic pathways or anaerobic pathways. Anaerobic pathways are up regulated at the beginning of all activity and do not use oxygen to synthesize cellular energy (39). However, the aerobic pathway is a high capacity energy producer that utilizes oxygen to combust fuels for energy (39). Fuel sources for energy metabolism are derived from three major sources, fats, carbohydrates, and proteins, each source acting in cooperation with the others to sustain bodily function. These macromolecules in combination with oxygen in the aerobic pathway are transported to the mitochondria, the power house of

the cell, to be combusted into usable energy in the form of adenosine triphosphate (ATP) (3).

Dependent upon cellular energy demands desired substrates will be selected to form ATP. Carbohydrates are the primary fuel source during intense exercise and there stored form as glycogen acts as a metabolic regulator for fatigue, while fats and proteins are the desired fuel sources at rest (39). Energy substrates travel within the blood stream until extraction or deposition occurs. Glucose can be extracted from the blood via insulin or by cellular glucose transporters (GLUT) (68). Fatty acids bind to carrier 
proteins in the blood, like albumin or even insulin, and are transported to the cell for utilization or storage (39). Fats and carbohydrates work cooperatively to maximize energy production. Fats are oxidized in a residual carbohydrate metabolism allocating glycogen sparing; therefore delaying the onset of fatigue (39). Protein degradation for ATP synthesis is only used in highly fasted states. All substrates can be combusted for the production of cellular energy or they can be stored within the body for later usage in common sites such as: skeletal muscle, adipose tissue, liver, and the brain. However, substrate utilization and storage are highly regulated by metabolic enzymes on a minute by minute basis.

\section{Regulation}

Storage and utilization of energy substrates is dependent upon cellular energy demand and subsequent metabolic regulating pathways. Adenosine monophosphate activated protein kinase (AMPK) acts as a local energy sensor and regulates mitochondrial biogenesis and overall metabolism through allosteric mechanisms $(8,16$, 40). Allosteric regulation of metabolism is controlled through AMPK based on a continuum of caloric intake from a fed state to a fasted state and on cellular energy levels meaning, when energy levels are low the AMPK pathway is activated and when energy levels are high AMPK activity declines. Peroxisome proliferator activated receptor gamma coactivator alpha (PGC-1alpha) and sirtuins one (SIRT1) are key signaling molecules that are allosterically mediated within the AMPK pathway that intrinsically regulates mitochondrial activity and mitochondrial biogenesis. In a fasted state the AMPK pathway is activated by the release of glucagon thereby stimulating SIRT1 via cAMP cascade, which deacetylates PGC-1alpha, increasing fatty acyl CoA 
dehydrogenase and carintine acyletransferase, driving mitochondrial biogenesis and oxidative capacity of long and short chain fatty acids $(1,16,50)$. PGC-1alpha is considered to be a "master regulator" of mitochondrial biogenesis in skeletal muscle and has been shown to up regulate fatty acid metabolism and energy expenditure within the mitochondria, as well as to improve insulin sensitivity in times of stress $(22,65)$. SIRT1 and PGC-1alpha acting in corporation enhance fatty acid metabolism by increasing the gene expression of metabolites used in oxidative metabolism, however, fat metabolism has been shown to be down regulated in SIRT1 knockout models citing the requirement of SIRT1 for mitochondrial oxidative capacity (22). Cytochrome c (cyt c) and citrate synthase (CS) are two mitochondrial molecular signalers that reside within the inner membranes of the mitochondria and electron transport chain (ETC) and dictate metabolic energy flow through electron acceptance during oxidative metabolism (39). When metabolic demand increases chronically, as with exercise training, mitochondria are stimulated for local division increasing mitochondrial density, which can be observed by expression of mitochondrial DNA (mtDNA). Regulation of metabolism can be affected by various external stimuli including exercise and diet. Diets high in fat leading to obesity have been shown to negatively affect enzymatic regulation and cause metabolic dysfunction.

\section{High fat diets}

Over the last 20 years, across all age groups, obesity rates have risen due to lack of daily physical activity and the over consumption of energy dense foods. In 2010 the United States had more than $1 / 3$ of all adult Americans classified as obese with a body mass index (BMI) greater than or equal to $30 \mathrm{~kg} / \mathrm{m} 2$ (48). The standard American diet 
consists of $52 \%$ carbohydrate, $15 \%$ protein, $33 \%$ fat with $12 \%$ coming from saturated fat, a ratio of 1.8 to 1 monounsaturated to polyunsaturated fat, and a ratio of 20 to 1 omega 6 to omega $3(23,26,61)$. In 2013 the trend for obesity continues as the amount of saturated fat intake increases. Saturated fats (SF), commonly referred to as palmitic and stearic acid, are known storage fats that are linked to dietary obesity and excessive adiposity. Foods high in SF include red meats, many dairy products like cheese and whole milk, pork, fast food and processed foods (69). SF's have been shown to instigate pro-inflammatory pathways via $\mathrm{NF}_{\mathrm{k}} \mathrm{B}$ and can cause insulin resistance and dysregulation in metabolic enzymes (34). Many side effects of diets high in saturated fat have been linked to fatty liver disease, diabetes, cardiovascular disease, hypertension, metabolic syndrome and many other health disparities.

Other types of HFD's include polyunsaturated fat diets (PUFA) and monounsaturated fat diets (MUFA). PUFA's, particularly omega-6 and omega-3, are related to healthy fat intake by consuming foods such as fish, soy products, and nuts (57). MUFA's are similar to PUFA's compositionally, but MUFA's can be obtained through oils such as canola and olive (23). MUFA's have been shown to attenuate the effects of a diet high in saturated fat, but negates normal metabolic activity when combined with high PUFA diets (23).

Researchers are now comparing similar diets in animal and human models to represent the population to determine how local diets are affecting energy metabolism. A predominate amount of calories are proportioned from fats to observe similar caloric intake patterns of the standard American to determine if a HFD has any effect on energy metabolism and substrate utilization. Diets consisting of 35-40\% fat are commonly used 
and have been shown to attenuate insulin sensitivity (41) therefore leading to declines in glycogen synthesis (10) and metabolism of fatty acids by PCG-1alpha stimulation (65).

Overall, it has been shown that excessive fat intake is linked to serious metabolic dysregulation in the body, with lipid accumulation causing oxidative stress within the cell leading to declines in glucose uptake, pre-empting fatigue $(58,60,71)$. Excessive lipid accumulation by a HFD over stimulates the cell causing increased reactive oxygen species (ROS), thereby causing oxidative stress and increasing inflammation potentially leading to dysfunctional enzymatic and molecular signaling activity. This phenomenon has been highly researched in skeletal muscle.

\section{High Fat Diet and Skeletal Muscle}

Skeletal muscle is the primary location for oxidative metabolism of glucose and lipid substrates, which also makes it a prime location to experience metabolic dysregulation during times of stress. HFD's can act as a metabolic stressor by inducing an overload on the body with excess lipid accumulation and altered signaling of AMPK $(1,19)$ causing localized oxidative stress and fatigue. Diets high in SF have been shown to impair insulin sensitivity and enzyme regulation by causing severe inflammation through the $\mathrm{NF}_{\mathrm{K}} \mathrm{B}$ pathway increasing inflammatory cytokines IL-6 and TNF-alpha (32, 33). Diets high in SF have also been shown to decrease PGC-1alpha activity thereby reducing oxidative phosphorylation of lipids $(11,34)$. AMPK and PGC-1alpha gene expression have been shown to increase with the acute manipulation of a HFD and concomitantly SIRT1 gene expression is up regulated to enhance fatty acid metabolism during times of metabolic stress, thereby increasing mitochondrial biogenesis driving energy metabolism in times of low demand $(1,19,22)$. 
However, long term manipulations of a HFD have shown the opposite effects on AMPK, with a decline in activity (24). Chronic ingestion of a high fat diet is well known to cause insulin insensitivity. With reduced glucose uptake by insulin, glycogen synthesis in skeletal muscle is reduced potentially leading to metabolic fatigue. Therefore, with chronic lipid accumulation both carbohydrate and lipid uptake are halted and subsequently reduced by obesity related insulin insensitivity; that may be correlated with inflammation $(27,51,68)$. Cleasby et al. 2011 has demonstrated with a five week HFD in mice, a reduction in both insulin sensitivity and glycogen synthesis in skeletal muscle (10). Sparks et al. 2006 found a similar response in a three week HFD study (62).

Previous research completed in our lab has shown that 16 weeks of a high fat diet with $12 \%$ saturated fat, the most similar to the standard American diet, shows the greatest amount of adiposity, inflammation, and subsequent metabolic dysregulation in peripheral tissues (17). Through extensive review of the literature it can be noted that the effects of a HFD on skeletal muscle metabolism and enzymatic regulation has been well researched, however, results have yet to be definitively addressed.

\section{Differences in Metabolic Function}

Oxidative phosphorylation has been shown in several studies to be affected by the manipulation of a HFD; some studies cite an increase in mitochondrial oxidative capacity with increased lipid availability, while others cite the reverse effects. Length of the manipulation, the amount of SF, the total amount of fat intake, and molecular makeup of fat are potential parameters affecting the dichotomous outcomes. Iossa, S. et al. 2002 demonstrated that two weeks of a HFD enhanced mitochondrial oxidative capacity utilizing lipids as the primary fuel source (30). Lionette, L. et al. 2007 and Hoeks, J. et al 
2008 observed similar findings in a seven and eight week HFD study respectively (29, 38). Lionette used 50\% energy intake from lipids with a PUFA to saturated fat ratio of 1.7 (38) and Hoeks used 47\% energy intake coming from lipids (29). However, longer manipulations of a high fat diet have elucidated the reverse effects. Enos et al. has shown that 16 weeks of a high fat diet with $40 \%$ energy intake coming from lipids and $12 \%$ saturated fat shows the greatest amount of adiposity and subsequent metabolic dysregulation, disparately saturated fat amounts independent of total fat shows the opposite effects with $24 \%$ saturated fat displaying the lowest levels of metabolic dysregulation and even normal mitochondrial function (17). Enos and colleges state a potential mechanism in molecular differences between long and short chain fatty acids. Gomes et al. completed a 12 week HFD study and found declines in mitochondrial enzymatic and molecular signaling activity, but used a similar diet to Lionette and colleges at $60 \%$ energy intake coming from lipids (24). This finding suggests that length of the HFD and chronic ingestion of lipids is the main contributor to metabolic syndrome. However, these dichotomous outcomes related to duration of study and amount of total fat and SF have not been thoroughly explained in the literature and future research is warranted.

In summary current research has shown with the manipulation of a HFD that regulation of AMPK becomes dysfunctional subsequently altering allosteric regulation of PGC-1alpha, SIRT1, and insulin decreasing homeostatic metabolic function in skeletal muscle. Excessive lipid accumulation causes oxidative stress on the cell affecting normal metabolism of fuel substrates. Chronically glucose and lipid substrate utilization and storage becomes impaired and mitochondrial biogenesis declines. There is copious 
evidence displaying the negative effects of a HFD on skeletal muscle metabolism, however, we have yet to elucidate how metabolism and mitochondrial biogenesis is affected with a HFD in the central regulator of our body, the brain.

\section{Brain}

The brain is the apogee of the CNS and is divided into white and grey matter in four primary lobes: occipital, temporal, frontal, and parietal, which are then divided into smaller subsections (40). The subsections are responsible for controlling complex processes involving motor function, decision making, metabolism, mood, information processing, and regulating energy and fatigue through neuronal communication (40). The medulla oblongata and pons are regions of the brain stem. Both regions control motor and sensory function in the body. The medulla oblongata specifically controls autonomic responses including respiratory, cardiovascular and digestive functioning (40). The hippocampus is part of the limbic system that involves memory storage and information retrieval (40). The cerebellum controls bodily movement; consciously and subconsciously (40). The hypothalamus is the central regulator of all metabolism controlling hunger and thirst in "feeding centers" and "satiety centers" with hormonal responses that can stimulate glucose and lipid uptake, utilization, and storage (6).

The brain utilizes and stores energy substrates to carry out basic daily processes. Contrary to skeletal muscle, the brain does not metabolize lipids as an energy substrate, but growing evidence sites the "sensing" of fatty acids to dictate hormonal signaling (36, 57). Glucose is the primary energy substrate in the brain that is utilized by neurons and stored in astrocytes, which accounts for $99 \%$ of all ATP production $(20,55)$. Astrocytes are connected to neurons by capillary networks that allow glucose to move freely for 
energy usage. Similar to skeletal muscle, the brain uses GLUT receptors, specifically GLUT1, 3, to transport glucose from the blood across the neuronal membrane for direct utilization by the neuron or storage in the astrocyte where glycogen is primarily localized $(14,15,55)$. Astrocytic glycogen is the only source of glycogen storage in the brain, but compared to skeletal muscle storage is insignificant (15).

The brain functions primarily under aerobic metabolism using mitochondria to oxidize glucose for energy due to the limited glycolytic capacity of the CNS $(9,43)$. Neuronal stimulation is activated via sodium release, coupled by glutamine reuptake, which stimulates glucose metabolism in the brain (55). Brain glucose metabolism in neurons is regulated via AMPK, which mediates molecular signaling of SIRT1 and PGC1alpha, thereby regulating insulin (74). AMPK also moderates nutrient usage and hormonal regulation $(4,42)$.

Hormone release, regulated by the hypothalamus, contributes stimulatory affects to the uptake, storage, and utilization of glucose under different physiological states. In a stressful physiological state, metabolic demand changes and redirects blood flow to high demand tissues. For example, during exercise blood flow is redistributed away from the gut to the skeletal muscle, the heart, skin, and the brain (39). Exercise training enforces chronic adaptations of increased delivery of oxygen and nutrients, oxidative capacity and mitochondrial biogenesis to accommodate the increasing metabolic demands (63). Post exercise, blood flow returns to trivial areas, but remains elevated in both skeletal muscle and brain at $20 \%$ and $14 \%$ respectively (39). Exercise is not the only physiological stressor that can induce changes in metabolic function and mitochondrial biogenesis; 
stress originating from the intake of a high fat diet also leads to serious metabolic disturbances.

\section{High fat diet and brain metabolism}

The CNS operates on glucose metabolism within the mitochondria with little to no oxidation of fatty acids. Despite this fact, research has shown that a diet high in fat can cause metabolic dysregulation not only in peripheral tissues, but central tissues as well. Memory deficits and cognitive impairments are the most commonly studied phenomenon related to brain function with a HFD. Alzheimer's and age related dementia are two areas of research that have been linked to diet induced obesity and oxidative stress $(43,70)$. The hippocampus has been highly researched in regards to memory deficits and age related dementia due to its primary function of memory retrieval, storage and information processing. A recent study suggests that memory deficits are correlated to a decline in SIRT1 gene expression in the hippocampus of HFD fed mice (28). A decline in SIRT1 may be indicative of reduced metabolic function and cellular energy production (28). Diets high in SF's and cholesterol have been demonstrated to reduce the integrity of the blood brain barrier and cause severe gliosis throughout the hippocampus (21).

Throughout the brain insulin sensitivity is relatively low compared to skeletal muscle; however, in the hypothalamus insulin sensitivity is high allowing mediation of systemic hormone regulation. Insulin activity via hypothalamic signaling allows fatty acids to be stored in a process called lipogenesis by down regulating the sympathetic nervous system output to adipose tissue (59). Hypothalamic injury in accordance with excess lipid accumulation will disrupt normal insulin activity and sensitivity and may 
eventually lead to over production of glucose through gluconeogenic pathways and subsequently type 2 diabetes (59).

Over nutrition by a HFD has been shown to induce mitochondrial dysfunction in hypothalamic neurons with a decline in central glucose sensitivity (49). Morphologic measurements of mitochondria post HFD have revealed mitochondrial swelling that negated mitochondrial membrane potential, especially during oxidative stress (54). This finding suggests that oxidative stress may be leading to mitochondrial dysfunction and a decline in energy metabolism preempting metabolic fatigue. Oxidative stress is also a primary factor to the induction of inflammation by diet induced obesity and the increase in fat cell size and number (5).

\section{High fat diet and inflammation}

HFD's have been shown to take weeks to incite inflammation and dysfunctional metabolic regulation in skeletal muscle, however, in the brain it can be observed within 24 hours, with neuronal injury and impairment in the hypothalamus within the first week (66). HFD's have been shown to cause an increase in adipose tissue, which provides a storage site for fat and acts as a production site for inflammatory cytokines such as interleukin 1 beta (IL-1 $\beta$ ) and tumor necrosis factor alpha (TNF-alpha) (18). As adipocytes grow in size and number with a HFD, inflammatory cytokine production progressively worsens provoking systemic inflammation. The CNS blocks inflammatory cytokines from entering the brain through the blood brain barrier making it immune from peripherally instigated inflammation. However, the brain activates various inflammatory 
transcription factors, increasing cytokines, in response to local and systemic inflammation (67).

TNF-alpha was one of the first major cytokines discovered within the inflammatory pathway. Its production from immune cells, skeletal muscle, and adipose tissue propagates a wide systemic response. Unlike other pro-inflammatory cytokines, TNF-alpha instigates obesity by acting as a potent activator of fatty acid release through the $\mathrm{NF}_{\mathrm{k}} \mathrm{B}$ pathway and it inhibits the initiation of receptor substrates for insulin signaling (18). Lack of response by insulin can lead to an accumulation of free fatty acids and glucose in the blood thereby reducing glycogen storage.

TNF-alpha is also mediated through IL-1 $\beta$, a known pro-inflammatory cytokine that stimulates the up regulation of other inflammatory cytokines through IL-1 receptor signaling thereby activating the $\mathrm{NF}_{\mathrm{k}} \mathrm{B}$ pathway (45). IL-1 $\beta$ has recently been shown in the literature to be associated with Alzheimer's disease progression and other neurological disorders by affecting brain astrocytes and neuronal communication (45). Thaler and colleges have shown an up regulation of IL-1 $\beta$ and TNF-alpha in response to diet induced obesity over 4 weeks (66). Once inflammatory cytokines have infiltrated a tissue the overwhelming response is to activate other cytokines to the area stimulating further progression of pro-inflammatory processes leading to destruction of a tissue.

Research has shown that chronic inflammation from HFD's causes oxidative stress instigating the increase in reactive oxygen species (ROS) $(2,7)$. ROS are common byproducts produced during metabolism, but can increase severely during times of oxidative stress and mitochondrial damage (39). ROS can also cause mutations in 
mtDNA inducing mitochondrial collapse (25). The inflammatory cascade may be involved in every aspect of metabolic oxidative stress due to the chronic inflammatory response with a HFD. Current research on HFD's has developed managerial treatments for reducing inflammation and fatigue, while increasing insulin sensitivity, but no formal cures have been discovered.

\section{Potential treatments}

Consuming a diet high in fat is well known to cause adiposity and low grade chronic inflammation. This inflammation may be the primary cause of insulin insensitivity in both the brain and skeletal muscle. HFD induced oxidative stress caused by excessive lipid accumulation has also contributed to what seems to be irreversible affects on energy metabolism. Improving quality of life and life longevity is the primary reason for research on human pathophysiology. Quercetin is a supplement that is found in many food sources such as fruits and vegetables and has been shown to work through anti-inflammatory pathways (13). Our lab has shown the effective use of Quercetin in enhancing brain and muscle mitochondrial biogenesis that stimulated increases in endurance capacity and voluntary activity in mice (13).

Resveratrol is similar to Quercetin in that it is found in common everyday foods like red wine and grapes and has been associated with elimination of free radicals and reversal of insulin resistance and diet induced obesity (75). Jeon et al. 2012 has shown that resveratrol can attenuate inflammation and improve memory deficit in obesity induced diabetic mice (31). Resveratrol has also been shown to increase neurogenesis and brain-derived neurotrophic factor in the hippocampus (44). 
More recently the health community has been trying to implement exercise as a prescription for improving health disparity rather than prescribing medication. The American College of Sports Medicine has incited "Exercise is Medicine" and continues to excite interest in prescribing personalized treatment through exercise. Exercise is multifunctional in that several modes exist: resistance training, swimming, running, biking, walking, tennis, baseball, etc. producing similar effects allowing subjects to choose the most enjoyed form available that is consistently overloading the body to elicit adaption. Currently it has been shown that chronic exercise training can attenuate obesity related insulin insensitivity by reducing excess lipid accumulation and enhancing signaling for glucose uptake and utilization in skeletal muscle (52).

\section{Conclusion}

In summation, a diet high in fat not only causes peripheral distress, but it also causes central dysregulation. The extent of central oxidative stress on metabolic function has yet to be fully elucidated. Morphologic observation on mitochondria and changes in insulin sensitivity with hypothalamic injury by HFD has been assessed; however, regulation of metabolism through enzymatic function has yet to be researched beyond SIRT1. Future research linking obesity to metabolic dysregulation, changes in mitochondrial biogenesis, and inflammation is warranted. 


\section{REFERENCES}

1. Abbot, M.J., Constantinescu, S., \& Turcotte, L.P. 2012. AMP-activated protein kinase $\alpha 2$ is an essential signal in the regulation of insulin-stimulated fatty acid uptake in control-fed and high-fat-fed mice. Experimental Physiology. 603-617.

2. Anderson EJ, Lustig, M.E.,Boyle, K.E., Woodlief, T.L., Kane, D.A., Lin, C.T., Price, 3rd J.W., Kang, L., Rabinovitch, P.S., Szeto, H.H., Houmard, J.A., Cortright, R.N., Wasserman, D.H., \& Neufer, P.D. 2009. Mitochondrial H2O2 emission and cellular redox state link excess fat intake to insulin resistance in both rodents and humans. Journal of Clinical Investigations. 119: 573-581.

3. Aw, T.Y. \& Jones, D.P. 1989. Nutrient supply and mitochondrial function. Annual Review of Nutrition. 9: 229-51.

4. Bantubungi, K., Prawitt, J. \& Staels, B. 2012. Control of metabolism by nutrientregulated nuclear receptors acting in the brain. Journal of Steroid Biochemistry and Molecular Biology. 130: 126-137.

5. Bassols, J., Botas, P., Moereno-Navarrete, J.M., Delgado, E., Oretga, F., Ricart, W. \& Fernandez-Real, J.M. 2010. Environmental and genetic factors influence the relationship between circulating IL-10 and obesity phenotypes. Epidemiology. 18: 611-618.

6. Brobeck, J.R. 1946. Mechanism of the development of obesity in animals with hypothalamic lesions. Physiology Review. 26(4):541-59.

7. Boudina, S., Sena, S., Sloan, C., Tebbi, A., Han, Y.H., O’Neil, B.T., Cooksey, R.C., Jones, D., Holland, W.L., McClain, D.A. \& Abel, E.D. 2012. Early mitochondrial adaptations in skeletal muscle to diet-induced obesity are strain dependent and determine oxidative stress and energy expenditure but not insulin sensitivity. Endocrinology. 153(6): 2677-2688.

8. Canto, C., Gerhart-Hines, Z., Fiege, J.N., Lagouge, M., Noriega, L., Milne, J.C., Elliot, P.J., Puigserver, P., \& Auwerx, J. 2009. AMPK regulates energy expenditure by modulating NAD1 metabolism and SIRT1. Activity and Nature. Vol. 45. 
9. Cardoso, S., Santos, R.X., Correia, S.C., Carvalho, C., Santos, M.S., Baldeiras, I., Oliveira, C.R. \& Moreira, P.I. 2013. Insulin induced recurrent hypoglycemia exacerbates diabetic brain mitochondrial dysfunction and oxidative imbalance. Neurobiology of Disease. 49: 1-12.

10. Cleasby, M.E., Lau, Q., Polkinghorne, E., Patel, S.A., Lesli, S.J., Turner, N., Cooney, G.J., Xu, A., \& Kraegen, E.W. 2011. The adaptor protein APPL1 increases glycogen accumulation in rat skeletal muscle through activation of the PI3-kinase signaling pathway. Journal of Endocrinology. 210. 81-92.

11. Coll, T., Jove, M., Rodriguez-Calvo, R., Eyre, E., Palomer, X., Sanchez, R.M., Merlos, M., Laguna, J.C. \& Vazquez-Carrera M. 2006. Palmitate-mediated downregulation of peroxisome proliferator-activated receptor-g coactivator 1 a in skeletal muscle cells involves MEK1/2 and nuclear factor-kB activation. Diabetes. 55: 2779-2787.

12. Dalsgaard, M.K. \& Secher, N.H. 2007. Mini-review: the brain at work: a cerebral metabolic manifestation of central fatigue. Journal of Neuroscience Research. 85:3334-3339.

13. Davis, J.M., Murphy, E.A., Carmichael, M.D. \& Davis, B. 2009. Quercetin increases brain and muscle mitochondrial biogenesis and exercise tolerance. American Journal of Physiology. 296. 1071-1077.

14. Deniel, G.A., Wang, R.Y. \& Cruz, N.F. 2002. Generalized sensory stimulation of conscious rats increases labeling of oxidative pathways of glucose metabolism when the brain glucose-oxygen uptake ratio rises. Journal of Cerebral Blood Flow and Metabolism. 22: 1490-1502.

15. Dong, J., Chen, X., Cui, M., Yu, X., Pang, Q. \& Sun, J. 2012. Beta 2 adrenergic receptor and astrocyte glucose metabolism. Journal of Molecular Neuroscience. 48: 456-463.

16. Draznin, B., Wang, C., Adochio, R., Leitner, J.W., \& Cornier, M.A. 2012. Effects of dietary macronutrient composition on AMPK and SIRT1 expression and activity in human skeletal muscle. Hormone and Metabolic Research. 44: 650-655.

17. Enos, R.T., Velazquez, K.T., Davis, J.M., McClellan, J.L., Day, S.D., Carnevale, K.A. \& Murphy, E.A. 2013. Influence of dietary saturated fat content on adiposity, macrophage behavior, inflammation, and metabolism: composition matters. Journal of Lipid Research. 54: 152-163.

18. Fernandez-Sanchez, A., Madrigal-Santillan, E., Bautista, M., Esquivel-Soto, J., Morales-Gonzalez, A., Esqivel-Chirino, C., Durante-Montiel, I., Sanchez-Rivera, G., Valadez-Vega, C. \& Morales-Gonzalez, J.A. 2011. Inflammation, oxidative stress, and obesity. International Journal of Molecular Sciences. 12: 3117-3132. 
19. Fillmore, N., Jacobs, D.L., Mills, D.B., Winder, W.W., \& Hancock, C.R. 2010. Chronic AMP-activated protein kinase activation and a high-fat diet have an additive effect on mitochondria in rat skeletal muscle. Journal of Applied Physiology. 109: 511-520.

20. Fox, P.T., Raichle, M.E., Mintun, M.A. \& Dence, C. 1988. Nonoxidative Glucose Consumption during Focal Physiologic Neural Activity. Science. 241: 462-464.

21. Freeman, L.R. \& Granholm, A.E. 2012. Vascular changes in rat hippocampus following a high saturated fat and cholesterol diet. Journal of Cerebral Blood Flow and Metabolism. 32: 643-653.

22. Gerhart-Hines, Z., Rodgers, J.T., Bare, O., Kim, C., Kim, S.H., Mostoslavsky, R., Alt, F.W., Wu, Z.D., \& Puigserver, P. 2007. Metabolic control of muscle mitochondrial function and fatty acid oxidation through SIRT1/PGC-1 alpha. EMBO Journal. 26: 1913-1923.

23. Gillingham, L.G., Harris-Janz, S., \& Jones, P. 2011. Dietary Monounsaturated Fatty Acids Are Protective Against Metabolic Syndrome and Cardiovascular Disease Risk Factors. Lipids. 46: 209-228.

24. Gomes, A.P., Duarte, F.V., Nunes, P., Hubbard, B.P., Teodoro, J.S., Varela, A.T., Jones, J.G., Sinclair, D.A., Palmeira, C.M., \& Rolo, A.P. 2012. Berberine protects against high fat diet-induced dysfunction in muscle mitochondria by inducing SIRT1-dependent mitochondrial biogenesis. Biochimica et Biophysica Acta. 1822: 185-195.

25. Green, D.R., Galluzzi, L. \& Kroemer, G. 2011. Mitochondria and the autophagyinflammation-cell-death axis in organismal aging. Science. 333: 1109-1112.

26. Grotto, D. \& Zied E. 2010. The Standard American Diet and its relationship to the health status of Americans. Nutrition Clinical Practice. 25:603-612.

27. Hansen, P.A., Han, D.H., Marshall, B.A., Note, L.A., Chen, M.M., Mueckler, M., \& Holloszy, J.O. 1998. A high fat diet impairs stimulation of glucose transport in muscle. Functional evaluation of potential mechanisms. Journal of Biology and Chemistry. 273: 26157-26163.

28. Heyward, F.D., Walton, R.G., Carle, M.S., Coleman, M.A., Garvey, W.T., Sweatt, J.D. 2012. Adult mice maintained on a high-fat diet exhibit object location memory deficits and reduced hippocampal SIRT1 gene expression. Neurobiology of Learning and Memory. 98: 25-32.

29. Hoeks, J., Briede, J.J., Vogel, J.D., Schaart, G., Nabben, M., Moonen-Kornips, E., Hesselink, M., Schwauren, P. 2008. Mitochondrial function, content and ROS 
production in rat skeletal muscle: Effect of high-fat feeding. FEBS Letters. 582: $510-516$.

30. Iossa, S., Mollica, M.P., Lionetti, L., Crescenzo, R., Botta, M. \& Liverini, G. 2002. Skeletal muscle oxidative capacity in rats fed high-fat diet. International Journal of Obesity. 26: 65-72.

31. Jeon, B.T., Jeong , E.A., Shin, H.J., Lee, Y., Lee, D.H., Kim, H.J., Kang, S.S., Cho, G.J., Choi, W.S. \& Roh, G.S. 2012. Resveratrol Attenuates ObesityAssociated Peripheral and Central Inflammation and Improves Memory Deficit in Mice Fed a High-Fat Diet. American Diabetes Association.

32. Jove, M., Planavila, A., Laguna, J.C. \& Vazquez-Carrera, M. 2005. Palmitate induced interleukin 6 production is mediated by protein kinase $C$ and nuclear-factor $k B$ activation and leads to glucose transporter 4 down-regulation in skeletal muscle cells. Endocrinology.146: 3087-95.

33. Jove, M., Planavila, A., Sanchez, R.M., Merlos, M., Laguna, J.C. \& VazquezCarrera, M. 2006. Palmitate induced tumor necrosis factor-a expression in C2C12 skeletal muscle cells by a mechanism involving protein kinase $C$ and nuclear factor-kB activation. Endocrinology. 147: 552-61.

34. Kennedy, A., Martinez, K., Chuang, C., La Point, K. \& McIntosh, M. 2009. Saturated fatty acid-mediated inflammation and insulin resistance in adipose tissue: mechanisms of action and implications. Journal of Nutrition. 139: 1-4.

35. Kong, J., Shepel, P.N., Holden, C.P., Mackiewicz, M., Pack, A.I. \& Geiger, J.D. 2002. Brain glycogen decreases with increased periods of wakefulness: implications for homeostatic drive to sleep. Journal of Neuroscience. 22: 55815587.

36. Lam, T.K.T., Schwartz, G.J. \& Rossetti, L. 2005. Hypothalamic sensing of fatty acids. Nature Neuroscience. 8: 579-584.

37. Libby P. 2002. Inflammation in atherosclerosis. Nature. 420: 868-874.

38. Lionetti, L., Mollica, M.P., Crescenzo, R., D Andrea, E., Ferraro, M., Bianco, F., Liverini, G. \& Iossa, S. 2007. Skeletal muscle subsarcolemmal mitochondrial dysfunction in high-fat fed rats exhibiting impaired glucose homeostasis. International Journal of Obesity London. 31: 1596-1604.

39. Lippincott, Williams, \& Wilkins. "ACSM's Advanced Exercise Physiology. American College of Sports Medicine". Baltimore, MD. 2006. 
40. Martini, F.H. "Fundamentals of Anatomy and Physiology". Ed. 7. Pearson Education Inc. San Francisco, CA. 2006.

41. Matsuo, T., Iwashita, S., Komuro, M., \& Suzuki, M. 1999. Effects of high fat diet intake on glucose uptake in central and peripheral tissues of non obese rats. Journal of Nutritional Science and Vitaminology. 45: 667-673.

42. Minokoshi, K., Alquien, T., Furukaa, N., Kim, Y.B., Lee, A., Xue, B., Mu, J., Foufelle, F., Ferre, P., Bimbaum, M.J., Stuck, B.J. \& Kahn, B.B. 2004. AMPkinase regulates food intake by responding to hormonal and nutrient signals in the hypothalamus. Nature. 428: 569-574.

43. Moreira, P.I., Carvalho, C., Zhu, X., Smith, M.A., \& Perry, G. 2010. Mitochondrial dysfunction is a trigger of alzheimer's disease pathophysiology. Biochimica et Biophysica Acta. 1802: 2-10.

44. Moriya, J., Chen, R., Yamakawa, J., Sasaki, K., Ishigaki, Y. \& Takahashi, T. 2011. Resveratrol improves hippocampal atrophy in chronic fatigue mice by enhancing neurogenesis and inhibiting apoptosis of granular cells. Biol. Pharm. Bull. 34: 354-359.

45. Moynagh, P.N. 2005. The interleukin-1 signaling pathway in astrocytes: a key contributor to inflammation in the brain. Journal of Anatomy. 207: 265-269

46. Nagao, K.Y. 2010. Medium-chain fatty acids: functional lipids for the prevention and treatment of the metabolic syndrome. Pharmacological Resistance. 61:208212.

47. Nguyen, D.M. \& El-Serag, H.B. The epidemiology of obesity. Gastroenterol Clinical North Am. 39:1-7.

48. Ogden, C.L., Carroll, M.D., Kit, B.K., \& Flegal, K.M. 2012. Prevelence of obesity and trends in the distribution of body mass index among U.S. adults, 1990-2010. American Medical Association. 307: 491-497.

49. Parton, L.E., Ye, C.P. \& Coppari, R. 2007. Glucose sensing by POMC neurons regulates glucose homeostasis and is impaired in obesity. Nature. 449: 228-232.

50. Pfluger, P.T., Herranz ,D., Velasco-Miguel, S., Serrano, M., \& Tschop, M.H. 2008. SIRT1 protects against high fat diet induced metabolic damage. National Academy of Science. 105: 9793-9798.

51. Pehlemen, T.L., Peters, S.J., Heigenhauser, G., \& Spriet, L.L. 2005. Enzymatic regulation of glucose disposal in human skeletal muscle after a high-fat, lowcarbohydrate diet. Journal of Applied Physiology. 98:100-107. 
52. Phielix, E., Ruth, M., Margriet, O.D., Sparks, L. \& Hoeks, J. 2012. High oxidative capacity due to chronic exercise training attenuates lipid-induced insulin resistance. Diabetes. 61: 2472-2478.

53. Pickup, J.C. 2004. Inflammation and activated innate immunity in the pathogenesis of type 2 diabetes. Diabetes Care. 27:813-823.

54. Pipatpiboon, N., Pratchayasakul, W., Chattipakorn, N. \& Chattipakorn, S. 2012. PPAR agonist improves neuronal insulin receptor function in hippocampus and brain mitochondria function in rats with insulin resistance induced by long term high-fat diets. Neuroendocrinology. 153: 329-338.

55. Prebil, M., Jensen, J., Zorec, R. \& Kreft, M. 2011. Astrocytes and energy metabolism. Archives of Physiology and Biochemistry. 117: 64-69.

56. Porta, C., Rimoldi, M., Raes, G., Brys, L., Ghezzi, P., et al. 2009. Tolerance and M2 (alternative) macrophages polarization are related processes orchestrated by P50 nuclear factor kappa B. Proceedings of the National Academy of Sciences of the United States of America. 106: 14978-14983.

57. Qi, K., Hall, M. \& Deckelbaum, R.J. 2002. Long-chain polyunsaturated fatty acid accretion in brain. Clinical Nutrition and Metabolism. 5: 133-138.

58. Richardson, D.K., Kashyap, S., Baja,J. M., Cusi. K., Mandarino, S.J. 2005. Lipid infusion decreases the expression of nuclear encoded mitochondrial genes and increases the expression of extracellular matrix genes in human skeletal muscle. Journal of Biology and Chemistry. 280: 10290-10297.

59. Scherer, T., Lindtner, C., Zielinski, E., O’Hare, J., Filatova, N. \& Buettner, C. 2012. Short term voluntary overfeeding disrupts brain insulin control of adipose tissue lipolysis. Journal of Biological Chemistry. 287: 33061-33069.

60. Shortreed, K.E., Krause, M.P., Huang, J.H., Dhanani, D., Moradi, J., Ceddia, R.B. \& Hawke, T.J. 2009. Muscle-specific adaptations, impaired oxidative capacity and maintenance of contractile function characterize diet-induced obese mouse skeletal muscle. Plos One. 4: 7293.

61. Simopoulos, A.P. 2008. The importance of the omega-6/omega-3 fatty acid ratio in cardiovascular disease and other chronic diseases. Experimental Biology and Medicine. 233: 674-688.

62. Sparks, L., Xie, H., Koza, R.A., Mynatt, R., Bray, G.A., \& Smith, S.T. 2006. High-fat/low-carbohydrate diets regulate glucose metabolism via a long-term transcriptional loop. Metabolism Clinical and Experimental. 55: 1457-1463. 
63. Steiner, J.L., Murphy, E.A., McClellan, J.L., Carmichael, M.D. \& Davis, J.M. 2011. Exercise increases mitochondrial biogenesis in the brain. Journal of Applied Physiology. 111: 1066-1071.

64. Swanson, R.A., Morton, M.M., Sagar, S.M., \& Sharp, F.R. 1992. Sensory stimulation induces local cerebral glycogenolysis: demonstration by autoradiography. Journal of Neuroscience. 51:451-461.

65. Tanaka, T., Yamamoto, J., Iwasakai, S., Asaba, H., Hamura, H., Ikeda, Y., Watanabe, M., Magoori, K., Ioka, R.X., Tachibana, K., Watanabe, Y., Uchiyama, Y., Sumi, K., Iguchi, H., Ito, S., Doi, T., Hamakubo, T., Naito, M., Auwerx, J., Yanagisawa, M., Kodama, T., \& Sakai, J. 2003. Activation of peroxisome proliferator-activated receptor induces fatty acid oxidation in skeletal muscle and attenuates metabolic syndrome. National Academy of Science. 100: 15924-15929.

66. Thaler, J.P., Yi, C., Schur, E.A., Guyenet, S.J., Hwang, B.H., et al. 2012. Obesity is associated with hypothalamic injury in rodents and humans. Journal of Clinical Investigation. 122: 153-162.

67. Thaler, J.P., Choi, S.J., Schwartz, M.W., \& Wisse, B.E. 2010. Hypothalamic inflammation and energy homeostasis: resolving the paradox. Frontiers in Neuroendocrinology. 31: 79-84.

68. Tsunoda, N., Cooke, D.W., Ikemoto, S., Maruyama, K., Takahashi, M., Lane, M.D., \& Ezaki, O. 1997. Regulated expression of $5^{*}$ deleted mouse GLUT4 minigenes in transgenic mice: effects of exercise training and high fat diet. Biochemical and Biophysical Research Communications. 239: 503-509.

69. USDA, Agricultural Research Service. "USDA National Nutrient Database for Standard Reference". 21. 2008.

70. Whitmer, R.A., Gunderson, E.P., Barrett-Connor, E., Quesenberry, C.P.J. \& Yaffe, K. 2005. Obesity in middle age and future risk of dementia: a 27 year longitudinal population based study. BMJ. 330:1360

71. Wit, N., Derrien, M., Bosch-Vermeulen, H., Oosterink, E., Keshtkar, S., Duval, C., Bosch, J., Kleerebezem, M., Muller, M. \& Meer, R. 2012. Saturated fat stimulates obesity and hepatic steatosis and affects gut microbiota composition by an enhanced overflow of dietary fat to the distal intestine. American Journal of Physiology. 303: 589-599.

72. Wu, A., Ying, Z., \& Gomez-Pinilla, F. 2006. Oxidative stress modulates Sir2a in rat hippocampus and cerebral cortex. European Journal of Neuroscience. 23: 2573-2580. 
73. Wyatt, S.B., Winters, K.P., Dubbert, P.M. 2006. Overweight and obesity: prevalence, consequences, and causes of a growing public health problem. American Journal of Medical Science. 331:166-174.

74. Yu, L. \& Yang, J. 2010. AMPK activated protein kinase mediates activity dependent regulation of peroxisome proliferator activated receptor gamma coactivator alpha and nuclear respiratory factor 1 expression in rat visual cortical neurons. Neuroscience. 169: 23-38.

75. Zhang, J., Chen, L., Zhang, J., Zeng, T., Li, H., Xiao, H., Deng, X. \& Hu, X. 2012. The protective effect of resveratrol on islet insulin secretion and morphology in mice on a high-fat diet. Diabetes Research and Clinical Practice. 97: 474-482. 